\title{
Three-dimensional water entry of a solid body: a particle image velocimetry study
}

\author{
Mohammad Jalalisendia ${ }^{\mathrm{a}}$, Steven J. Osma ${ }^{\mathrm{a}}$, Maurizio Porfiri ${ }^{\mathrm{a}, *}$ \\ ${ }^{a}$ Department of Mechanical and Aerospace Engineering \\ New York University Polytechnic School of Engineering, Six MetroTech Center, Brooklyn, NY 11201, USA
}

\begin{abstract}
Understanding and predicting the hydrodynamic loading experienced by a solid body during water impact is critical for researchers and practitioners in naval engineering. While two-dimensional (2D) water entry problems have been extensively investigated, experimental data on 3D fluid-structure interactions during water impact are rather limited. Here, particle image velocimetry (PIV) is utilized to study the free fall vertical impact of a solid body, modeling a ship hull, on an otherwise quiescent fluid. Planar PIV is used to measure the velocity field on multiple cross-sections along the length and width of the model. These data are combined to infer the 3D velocity field in the entire fluid. The 3D velocity field is then utilized to reconstruct the pressure field by integrating the incompressible 3D NavierStokes equations in a time-varying domain, where both the free surface and the fluid-solid interface evolve in time. By evaluating the pressure field on the wetted surface of the model, we estimate the hydrodynamic loading during water entry. Experimental results demonstrate the central role of 3D effects on both the flow physics and the hydrodynamic loading. As the cross-sectional velocity decreases away from the mid-span, we observe a robust increase in the axial velocity component. This translates into a complex spatio-temporal dependence of the hydrodynamic loading, which is initially maximized in the vicinity of the pile-up and later increases toward the keel. Due to the deceleration of the model during the impact and the increase in the wetted surface, the hydrodynamic loading close to the mid-span in the early stage of the impact is considerably larger than the ends. The 3D flow physics is used to study the energy imparted to the fluid during the impact, which we find to be almost entirely transferred to the risen water, consisting of the pile-up region and the spray jet. Our methodology can be implemented for the analysis of other solid bodies with multiple geometric curvatures, and our experimental results can be utilized for the validation of 3D mathematical models of water entry.
\end{abstract}

Keywords: hydrodynamic loading, particle image velocimetry, pile-up, slamming, water impact

\section{Introduction}

The characterization of the hydrodynamic loading experienced by solid bodies during water entry is of fundamental importance for the design of naval structures (Faltinsen, 1990; Abrate, 2013). Planing vessels are continuously subject to impulsive loading during sailing and maneuvering, largely affecting their performance and survivability (Gu and Moan, 2002; Faltinsen et al., 2004; Hughes et al., 2013). Despite the geometric complexity of marine vessels, the vast majority of the technical literature has focused on two-dimensional (2D) water entry problems (Abrate, 2013).

By studying 2D water impacts, a number of tractable mathematical models has been developed to predict the hydrodynamic loading experienced by rigid (Von Karman, 1929; Wagner, 1932; Korobkin, 2004) and flexible (Faltinsen, 1997; Qin and Batra, 2009; Khabakhpasheva and Korobkin, 2013; Shams and Porfiri, 2015) structures, along with an array of computational techniques to detail the flow physics during the impact (Zhao and Faltinsen, 1993;

\footnotetext{
${ }^{*}$ Corresponding author. Tel.: +1-718-260-3681. Fax: +1-718-260-3532. Porfiri)

Email addresses: mj1283@nyu.edu (Mohammad Jalalisendi), sjo280@nyu.edu (Steven J. Osma), mporfiri@nyu.edu (Maurizio
} 
Battistin and Iafrati, 2004; Stenius et al., 2011). These theoretical studies have been supported by experimental efforts on laboratory-scale models approximating 2D geometries, which have helped isolating the role of the deadrise angle (Chuang, 1966; Tveitnes et al., 2008), heel angle (Shams et al., 2015; Judge et al., 2004), impact velocity (Panciroli and Porfiri, 2013; Tveitnes et al., 2008), geometric curvature (Panciroli et al., 2015; El Malki Alaoui et al., 2012), and structural flexibility (Panciroli and Porfiri, 2015; Stenius et al., 2013).

While these efforts have contributed to an improved understanding of the physics of water impact, the role of 3D phenomena has yet to be fully elucidated. Korobkin and Pukhnachov (1988) have studied the initial stage of the impact of a 3D body on an ideal fluid. Korobkin (2002) has proposed an exact solution for the water entry of an elliptic paraboloid within the framework of Wagner approximation (Wagner, 1932), and additional analytical developments of the framework were presented in Scolan and Korobkin (2001). A further improvement of this solution was proposed in Korobkin (2005), where the modified Logvinovich model (MLM) proposed in Korobkin (2004) was extended to 3D problems to account for nonlinear effects and the geometry of the impacting body. Therein, model predictions for the resultant force were compared with experimental data for an elliptic paraboloid. Scolan (2004) derived a semi-analytical formulation for the hydroelastic impact of a flexible conical shell.

Complementary to these analytical studies, 3D water entry problems have been computationally investigated by Gazzola et al. (2005); Tassin et al. (2012); Sun and Wu (2013). Specifically, Gazzola et al. (2005) numerically solved a linearized Wagner problem using variational inequalities and validated theoretical predictions against previous findings on a cone (Scolan, 2004) and elliptic paraboloid (Scolan and Korobkin, 2001). Tassin et al. (2012) developed a fast numerical scheme based on Wagner theory and boundary element method. The hydrodynamic force was calculated for three cases of an elliptic paraboloid, a wedge with conical ends, and a square pyramid. Force data were compared with experimental results demonstrating a very close agreement with experimental findings. Oblique water entry of a cone was studied in Sun and Wu (2013), using a boundary element method to solve a potential problem with fully nonlinear boundary conditions imposed on the body and free surface.

Beyond the measurement of the total force during water entry (Tassin et al., 2012), few efforts have sought to evaluate the distributed hydrodynamic loading (Battley and Allen, 2012; Jalalisendi et al., 2015; El Malki Alaoui et al., 2015). Battley and Allen (2012) explored 3D hydrodynamic loading on rigid and deformable wedges entering the water surface at a constant speed, by performing local pressure measurements on select locations on the impacting body. However, 3D effects could not be fully characterized due to the lack of spatially resolved data for the pressure. Using a similar approach, El Malki Alaoui et al. (2015) have recently investigated the impact of a pyramid entering the water surface at a constant velocity. Data from four independent pressure transducers were used to assess the self-similarity of the impact and quantify the role of the entry velocity.

In Jalalisendi et al. (2015), we have proposed an alternative approach for the detailed analysis of 3D water entry without the use of pressure transducers installed on the impacting body. This approach entails the measurement of the flow physics in the vicinity of the body using particle image velocimetry (PIV) (Raffel et al., 2007) and the subsequent reconstruction of the pressure field using Navier-Stokes equations (Van Oudheusden, 2013). For a wedge of square base impacting the water in free fall, we have demonstrated that 3D effects have a secondary role on the hydrodynamic loading experienced by the wedge. Specifically, we have found that only the cross-sections proximal to the edges of the specimen are affected by the axial flow along the length of the wedge.

In this work, we seek to extend the analysis to a more complex geometry described by multiple curvatures that result in a highly 3D flow physics. Reconstructing the hydrodynamic loading in the vicinity of a complex shaped model require significant PIV advancements to isolate the flow field in the 3D pile-up region, where the pressure is expected to be maximized (Korobkin, 2004; Tassin et al., 2012). Different from the wedge considered in Jalalisendi et al. (2015), the cross-sections of the model used in this study vary along its axis, causing them to asynchronously impact the water surface.

Our approach is grounded on recent efforts on the use of PIV to study 2D water entry problems (Nila et al., 2013; Panciroli and Porfiri, 2013). Nila et al. (2013) and Panciroli and Porfiri (2013) independently demonstrated the feasibility of indirectly measuring the hydrodynamic loading on a rigid wedge from planar PIV measurements using different computational schemes. Following these initial efforts, additional studies were performed to elucidate the effect of the heel angle on the asymmetric water entry of rigid wedges Shams et al. (2015); investigate the role of geometric curvature and quantify the energy transfer between the impacting body and the water Panciroli et al. (2015); analyze the influence of structural flexibility Panciroli and Porfiri (2015); and validate the PIV-based pressure reconstruction on synthetic datasets to assess the contribution of several parameters for PIV analysis Facci et al. 
(2015).

The main objective of this work is to extend the 3D PIV-based pressure reconstruction technique of Jalalisendi et al. (2015) to study water entry of bodies with a complex geometry, representative of marine vessels (Faltinsen, 2006). The shape of the model is designed to proxy a miniature ship hull. The model enters the water surface with pure vertical velocity, dropping from a height of $50 \mathrm{~cm}$ in free fall. We utilize a planar PIV system to measure flow field on several cross-sections along both the length and width of the model. These velocity data are combined utilizing an interpolation scheme that outputs the 3D velocity field from 2D PIV measurements. We reconstruct the pressure field in the fluid through the methodology proposed in Jalalisendi et al. (2015), which entails the integration of Navier-Stokes equations in 3D. These data are used to characterize the hydrodynamic loading on the model and study the energy transferred to the fluid during impact.

The main contributions of this study are: (i) extending the experimental methodology developed in Jalalisendi et al. (2015) to resolve the flow physics in the pile-up for a model with multiple geometric curvatures; (ii) generalizing the analysis of Panciroli et al. (2015) to three dimensions to elucidate the energy imparted to the fluid during the impact and isolate the contribution to the risen water, including the pile-up and the spray jet; (iii) resolving the complex spatio-temporal dependence of the hydrodynamic loading experienced by the model during water entry; and (iv) quantifying the role of the axial flow along the length of the model on the hydrodynamic loading.

The rest of the paper is organized as follows. The experimental setup, data acquisition system, and experimental conditions are described in Sec. 2. In Sec. 3, we detail the PIV analysis along with our approach to measure the 3D velocity and pressure fields. In Sec. 4, we present the main results of the experiments, focusing on the pile-up, energy transfer, and hydrodynamic loading. Section 5 summarizes our findings and highlights the main conclusion of our work.

\section{Materials and methods}

\subsection{Experimental setup}

The experimental setup is a custom-built apparatus for the analysis of water entry of miniature vessels; further details of can be found in Panciroli and Porfiri (2013) and Panciroli et al. (2015). The setup is shown in Fig. 1 and consists of a $800 \times 230 \times 350 \mathrm{~mm}^{3}$ tank fixed on an aluminum frame holding the water tank $1.55 \mathrm{~m}$ above the floor. The impacting body is rigidly attached to a sledge that runs along two $1.5 \mathrm{~m}$ long rails before vertically impacting the water surface. All the experiments are performed at room temperature.

The dimensions of the model used in the experiments are illustrated in Fig. 2(a). The length, width, and height are 20,16 , and $5.6 \mathrm{~cm}$, respectively. The model is fabricated of an Acrylonitrile butadiene styrene (ABS) plastic printed in a Stratasys Dimension Elite rapid prototyping machine. The total dry mass of the falling body is $M_{t}=0.601 \mathrm{~kg}$. As shown in Fig. 2(a), the $X$ and $Y$-axes are along the model width and height, and the $Z$-axis is along the length, so that the $X Y$ - and $Y Z$-planes are both planes of symmetry ${ }^{1}$. The origin of the coordinate system is at the lowest point of the model, where it will first touch the water surface during the impact, see Figs. 2(a). The model is designed to proxy the complex shape of a ship hull, with varying curvatures in both the $X Y$ - and $Y Z$-planes. The profiles of the cross-sections along the model length are illustrated in Fig. 2(b); due to symmetry, the cross-sections are only presented for a quarter of the body. Using the notation in Panciroli et al. (2015), for any value of $Z$, the profile of the body can be written as

$$
Y(X, Z)=R_{Z}\left(\cos \beta_{Z}-\sqrt{1-\left(\frac{X}{R_{Z}}+\sin \beta_{Z}\right)^{2}}\right)+h_{Z}, \quad 0 \leq X \leq d_{Z}
$$

Here, $R_{Z}$ is related to the radius of curvature at a specific location $Z$ in the $X Y$-plane. Similarly, $\beta_{Z}$ is the deadrise angle at $Z, h_{Z}$ offsets the $Y$ coordinate, and $d_{Z}$ defines the range of $X$. All these functions can be well represented by a polynomial of order 6 of the form $a_{0}+a_{1} \frac{Z}{L}+a_{2}\left(\frac{Z}{L}\right)^{2}+a_{3}\left(\frac{Z}{L}\right)^{3}+a_{4}\left(\frac{Z}{L}\right)^{4}+a_{5}\left(\frac{Z}{L}\right)^{5}+a_{6}\left(\frac{Z}{L}\right)^{6}$. Table 1 summarizes the values of the coefficients for all the parameters, where $R_{Z}, d_{Z}$, and $d_{Z}$ are scaled by $L$.

\footnotetext{
${ }^{1}$ In naval hydrodynamics (Faltinsen, 2006), planning vessels are often described with the $X$-axis being along the length of the vessel (towards the bow), the $Y$-axis along the lateral direction of the vessel, and the $Z$-axis being in vertical direction.
} 


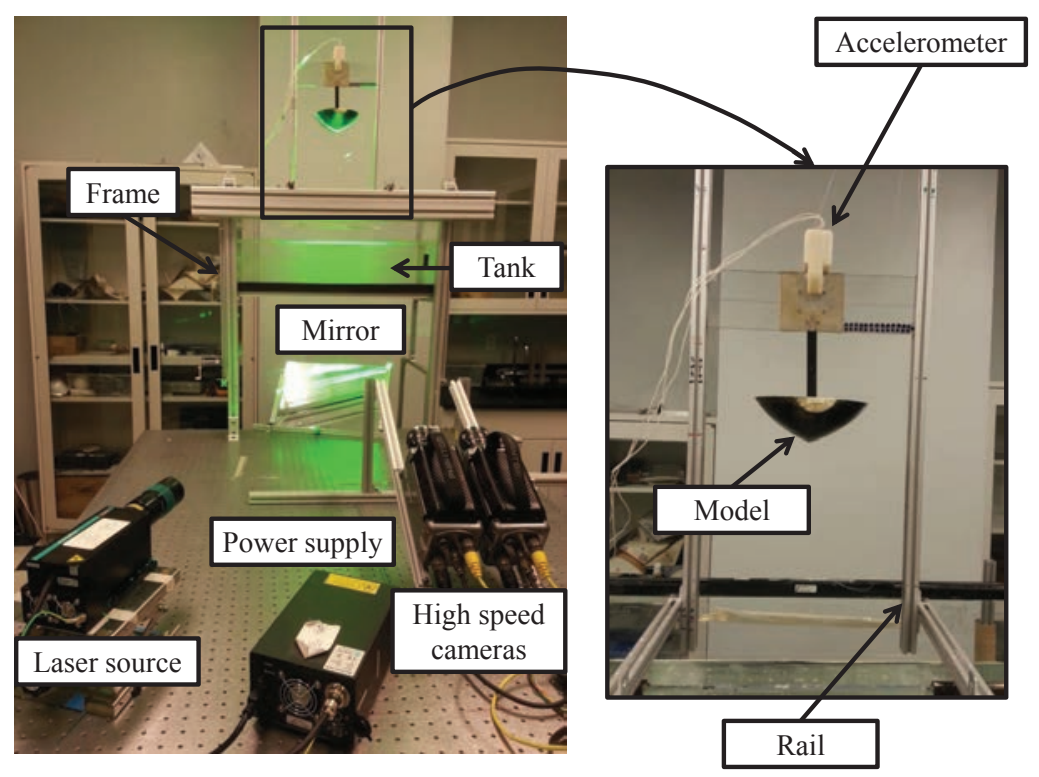

Figure 1: Image of the experimental setup with overlaid nomenclature of all components.

Table 1: Coefficients of the polynomial representation of $R_{Z} / L, h_{Z} / L, d_{Z} / L$, and $\beta_{Z}$.

\begin{tabular}{ccccc}
\hline coefficients & $R_{Z} / L$ & $h_{Z} / L$ & $d_{Z} / L$ & $\beta_{Z}[\mathrm{rad}]$ \\
\hline \hline$a_{0}$ & $8.351 \times 10^{-1}$ & 0 & 0.400 & $3.142 \times 10^{-1}$ \\
$a_{1}$ & $-15.932 \times 10^{-1}$ & $28.434 \times 10^{-3}$ & $-86.702 \times 10^{-3}$ & $-5.248 \times 10^{-1}$ \\
$a_{2}$ & $89.444 \times 10^{-1}$ & $8.896 \times 10^{-1}$ & $-29.822 \times 10^{-3}$ & $24.248 \times 10^{-1}$ \\
$a_{3}$ & -64.804 & $-25.397 \times 10^{-1}$ & $-65.216 \times 10^{-1}$ & $-94.533 \times 10^{-1}$ \\
$a_{4}$ & -219.912 & -3.017 & 40.669 & -13.805 \\
$a_{5}$ & -364.640 & 33.454 & -107.734 & 104.563 \\
$a_{6}$ & 231.302 & -37.347 & 90.630 & -118.984 \\
\hline
\end{tabular}

\subsection{Data acquisition system}

A time-resolved planar PIV system is utilized to measure 2D velocity fields in multiple cross-sections of the model and, ultimately, reconstruct the complete 3D velocity field. The PIV system consists of two high speed Phantom cameras V.9.1 and a 5W ND:YAG Ray Power laser source, see Fig. 1. Silver-coated hollow glass spheres with a mean diameter of $44 \mu \mathrm{m}$ are used as particle tracers. As shown in Fig. 1, the particles are illuminated at the measurement plane by reflecting the laser sheet from the laser source using a mirror mounted under the tank.

Due to the complex shape of the body and the resulting 3D free surface elevation, aberrations at the air-water interface hinder the possibility of using the configuration in Jalalisendi et al. (2015). Thus, the high speed cameras are mounted on a custom-made frame to record the impact at an angle of $27^{\circ}$ with respect to the optical table, see Fig. 1. The cameras are synchronized and positioned such that they image two contiguous regions of the fluid with an acquisition frequency of $4 \mathrm{kHz}$, which corresponds to a $0.25 \mathrm{~ms}$ time step. The selection of the sampling frequency is based on practical constraints and methodological issues, associated with planar PIV (Raffel et al., 2007). The total acquisition time is $18 \mathrm{~ms}$ starting from the instant when the model touches the water surface in that particular measurement plane. This time window corresponds to 73 frames per trial at each measurement location. DynamicStudio software package is used for camera synchronization, image acquisition, and storage.

In general, such a configuration may induce image distortions due to the cameras' angle with respect to the plane of the laser sheet (Lin and Perlin, 1998). In order to dismiss this possibility, we acquire images of a calibration target. 


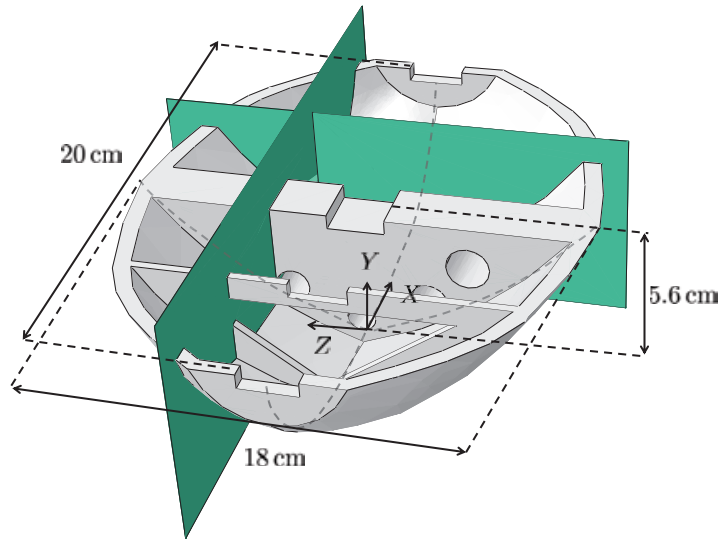

(a)

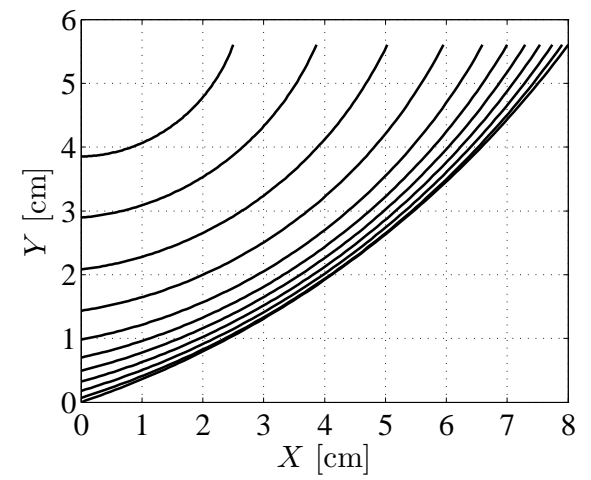

(b)

Figure 2: (a) Computer aided design of the model with overlaid dimensions and representative laser planes for cross-sectional and axial PIV. (b) Cross-section profiles of the model. Cross-sections are plotted for different values of $Z$ from the mid-span $(Z=0 \mathrm{~cm})$ to the end $(Z=10 \mathrm{~cm})$ of the body.

For simplicity, only one camera at a resolution of $688 \times 552 \mathrm{pixel}^{2}$ is used for calibration. The camera is angled at $27^{\circ}$ with respect to the optical table, as in the actual experimental setup shown in Fig. 1, and the target, with a reference grid size of $8 \times 8 \mathrm{~mm}^{2}$, is systematically rotated about the two axis defining the PIV measurement plane. We consider 9 different configurations by varying the two rotation angles from $0^{\circ}$ to approximately $20^{\circ}$ and $30^{\circ}$ (both clockwise and counterclockwise) with a step size of $10^{\circ}$ and $15^{\circ}$ about the horizontal and vertical axes, respectively. These images are then utilized for calibration using the Matlab camera calibration toolbox that outputs the complete distortion model parameters. This model accounts for both the radial and tangential distortions (Heikkila and Silvén, 1997). Our analysis suggests that the camera's angle with respect to the optical table induces a maximum distortion in the field of view of at most 2 pixels. Such distortion tends to be more pronounced in the bottom part of the image, which is the least affected by the entry of the model (Panciroli and Porfiri, 2013). Thus, the effect of distortions is not considered in the PIV analysis.

For each PIV measurement plane, the same calibration target is then used to calibrate the acquisitions from the two cameras. The calibration target is also utilized to identify the overlapping region between the cameras and to merge the images into a single image using a Matlab script. The overall resolution of the images after merging are $1149 \times 551$ pixel $^{2}$, corresponding to $230 \times 110 \mathrm{~mm}^{2}$ with 4.99 pixels $/ \mathrm{mm}$.

An 805M1-200 accelerometer manufactured by Measurement Specialties Inc. with a range of $\pm 1962 \mathrm{~m} / \mathrm{s}^{2}$ is utilized to estimate the acceleration of the falling body. The accelerometer is rigidly mounted on top of the sledge using an ad-hoc holder, as shown in Fig. 1.

\subsection{Experimental conditions}

To reconstruct the 3D velocity field from planar PIV, we adapt the methodology we have proposed in Jalalisendi et al. (2015) for the analysis of 3D water entry problems. A fixed Cartesian coordinate system is selected, with the $x, y$, and $z$-directions along the width, height, and length of the model, respectively, and the origin on the undisturbed water surface, at the center of the model. Two sets of PIV measurements are conducted. In the first set, called crosssectional PIV, we measure the velocity components $u$ and $v$ in $x$ and $y$-directions for several values of $z$. In the second set, called axial PIV, the axial velocity component $w$ in $z$-direction and $v$ are measured for several values of $x$, see Fig 2(a).

Due to the symmetry of the impact, PIV measurements are performed on only a quarter of the model. In crosssectional PIV experiments, 11 locations are considered from the mid-span $(z=0 \mathrm{~cm})$ to the end $(z=10 \mathrm{~cm})$ of the model, with an equal step size of $1 \mathrm{~cm}$. Similarly, in axial PIV experiments, 9 equally spaced locations are selected from the keel $(x=0 \mathrm{~cm})$ to the edge $(x=9 \mathrm{~cm})$ with step size of $1 \mathrm{~cm}$. For axial PIV, the model is rotated $90^{\circ}$ about the $y$-axis to avoid moving the acquisition system. 
Experiments are conducted for an impact height of $50 \mathrm{~cm}$ under laboratory illumination conditions. For each measurement location in axial and cross-sectional PIV, we perform three repetitions totaling 60 independent drop tests. As we change the measurement plane by varying the location of the laser sheet, we also move the cameras to maintain a constant field of view (Jalalisendi et al., 2015).

An independent set of drop tests consisting of three repetitions is also executed to accurately measure the entry depth of the lowest point on the model $(X=Y=Z=0)$ with respect to the free surface $\xi$ and the acceleration $\ddot{\xi}$. The former is measured by tracking the displacement of 5 markers drawn on the sledge in the images, while the latter is directly quantified using the accelerometer. Different from the PIV experiments, a single camera with a resolution of $688 \times 552$ pixel $^{2}$ and $4 \mathrm{kHz}$ acquisition frequency is used in the study. To facilitate the tracking, extra lighting is used to illuminate the sledge and the camera is focused on the sledge instead of the body or the fluid.

\section{Data analysis}

\subsection{Image analysis}

PIV analyses are performed on the acquired set of images via the open-source Matlab graphical user interface "PIVlab" (Thielicke and Stamhuis, 2014a,b). The PIV settings are selected as follows: (i) fast Fourier transform multigrid scheme with decreasing interrogation window size of $64 \times 64,32 \times 32$, and $16 \times 16$ pixels (Scarano and Riethmuller, 1999, 2000); (ii) interrogation window overlap of 50\%; and (iii) $2 \times 3$ Gaussian subpixel interpolation (Nobach et al., 2005). The output of the PIV analysis is a temporal sequence of uniformly spaced velocity grid vectors for each trial.

The identification of the water free surface and the body surface is central to this analysis. Therefore, an image mask is placed on each frame to exclude the area above the free surface and the body from the fluid (Panciroli and Porfiri, 2013; Jalalisendi et al., 2015). The masking procedure is performed manually one frame at a time for the first repetition of each test in cross-sectional and axial PIV and then applied to the other two remaining repetitions. The masking data is also utilized to identify the location of the root of the water jet, corresponding to the last wetted point of the body in each PIV measurement plane.

The entry depth $\xi$ is estimated by analyzing the images via the Xcitex ProAnalyst software. The entry depth for each trial, calculated from the initial water surface, is the average displacement of 5 markers. The entry depth and acceleration are averaged across the three repetitions to compute $\xi$ and $\ddot{\xi}$. Second order finite difference scheme is utilized to estimate the speed of the model from the averaged entry depth data. Figure 3 shows $\xi$, $\dot{\xi}$, and $\ddot{\xi}$ as functions of time. These quantities are used in what follows for scaling salient kinematic and kinetic variables. In Fig. 3(c), when the wedge touches the free surface at $t=0 \mathrm{~ms}$, the acceleration starts from a negative value corresponding to gravity.

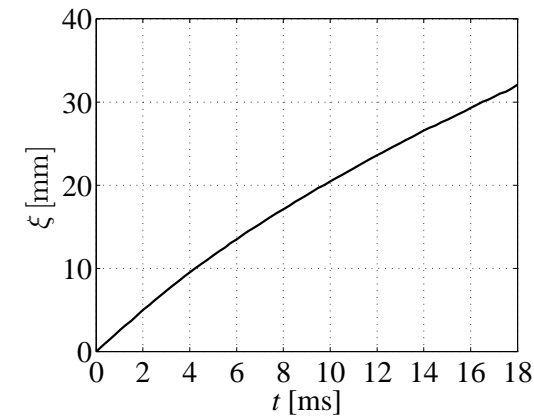

(a)

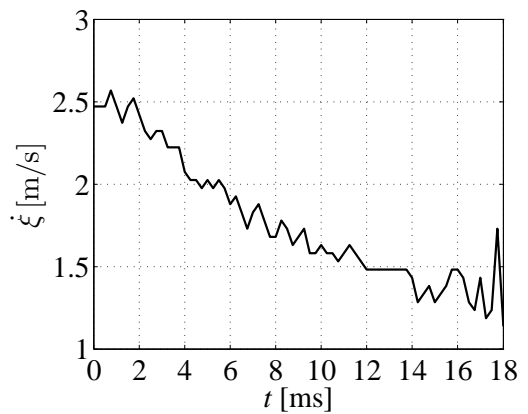

(b)

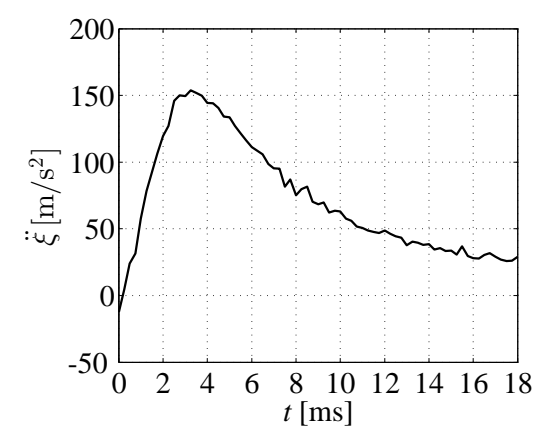

(c)

Figure 3: (a) Entry depth, (b) speed, and (c) acceleration of the impacting model as functions of time. Note that by integrating the acceleration data and using an initial velocity of $2.47 \mathrm{~m} / \mathrm{s}$ we obtain a highly comparable velocity time trace to Figure 3(b). 


\section{2. $3 D$ velocity and pressure reconstruction}

The cross-sectional and axial sets of PIV measurements are combined to obtain the 3D velocity field in the fluid. Specifically, we utilize the whole axial PIV dataset and the time histories of the water jet root location at each crosssectional PIV plane to enrich each cross-sectional PIV dataset with an estimation of the axial velocity component at every grid point. To this aim, we extend the interpolation scheme introduced in Jalalisendi et al. (2015) to account for a general impacting body shape. The interpolation method consists of the following steps:

(i) axial PIV data for each measurement location $x$ are averaged across the three repetitions to estimate the axial velocity component $w$ as a function of $y$ and $z$

(ii) time is set to zero when the keel touches the water surface at $z=0 \mathrm{~cm}$. The specific time instants at which the water wets the model in the other cross-sectional PIV measurement, $z \neq 0 \mathrm{~cm}$, are determined from the time history of the wetted length in the axial PIV experiment at $x=0 \mathrm{~cm}$ (henceforth referred to as time delays);

(iii) similarly, the time history of the wetted width at $z=0 \mathrm{~cm}$ from cross-sectional PIV experiments is used to determine the specific time instants when the water wets the model in the other axial PIV measurement planes, $x \neq 0 \mathrm{~cm}$;

(iv) the information obtained in steps (ii) and (iii) is used to associate the frames of each cross-sectional PIV experiments with the corresponding axial PIV frame;

(v) after completing the frame association, the planar velocity field for each of the 11 values of $z$ in cross-sectional PIV is enriched through the inclusion of the out-of-plane velocity component $w$. The velocity grid points overlapping in cross-sectional and axial PIV are identified and enriched with the axial velocity component $w$ calculated in step (i). The enrichment can only be performed for a small subset of grid points, dictated by the number of axial PIV locations;

(vi) a cubic spline interpolation scheme available in Matlab "curve fitting" toolbox is used to interpolate $w$ in the entire grid for each of the 11 values of $z$ in cross-section PIV; and

(vii) finally, steps (v) and (vi) are repeated for each frame to reconstruct the 3D velocity field for the whole duration of the impact.

Due to the use of the same image masking and the consistent field of view, the grid does not vary with the measurement location and the trials. Figure 5 summarizes the interpolation steps (ii), (iii), (iv), and (v). 3D velocity fields are finally smoothed using the multidimensional spline smoothing technique presented in Garcia (2010). Figure 4 displays the time delay in the model cross-section to touch the water surface with respect to the mid-span, determined from the axial PIV experiment at $x=0$. As shown in Fig. 4, locations far away from the mid-axis reach the water surface after several milliseconds from the keel impact.

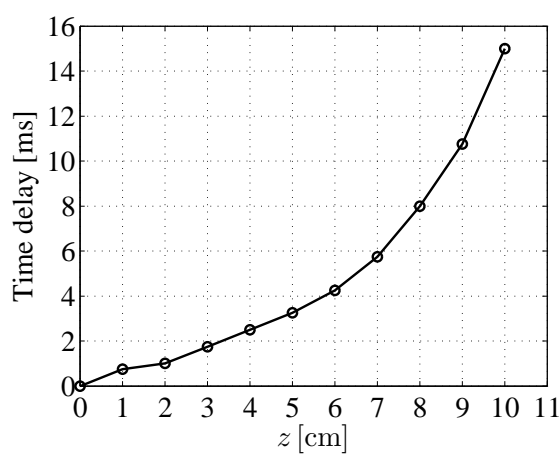

Figure 4: Time delay for each cross-sectional PIV location to touch the water surface. The linear interpolation (solid line) is only presented for ease of illustration. 
The pressure field reconstruction is based on the same approach proposed in Jalalisendi et al. (2015), which is an extension of the technique used in Panciroli and Porfiri (2013) to reconstruct the 2D pressure field using NavierStokes equations. Assuming the fluid to be incompressible and neglecting viscosity, for each value of $z$ Navier-Stokes equations read (Panton, 1994)

$$
\begin{aligned}
\frac{\partial p(x, y, z, t)}{\partial x}= & -\rho\left(\frac{\partial u(x, y, z, t)}{\partial t}+u(x, y, z, t) \frac{\partial u(x, y, z, t)}{\partial x}+v(x, y, z, t) \frac{\partial u(x, y, z, t)}{\partial y}+w(x, y, z, t) \frac{\partial u(x, y, z, t)}{\partial z}\right) \\
\frac{\partial p(x, y, z, t)}{\partial y}= & -\rho\left(\frac{\partial v(x, y, z, t)}{\partial t}+u(x, y, z, t) \frac{\partial v(x, y, z, t)}{\partial x}+v(x, y, z, t) \frac{\partial v(x, y, z, t)}{\partial y}+w(x, y, z, t) \frac{\partial v(x, y, z, t)}{\partial z}\right) \\
& -\rho g
\end{aligned}
$$

where $t$ represents time, $\rho$ is the constant density of the fluid ( $\rho=1000 \mathrm{~kg} / \mathrm{m}^{3}$ in our analysis), and $g=9.81 \mathrm{~m} / \mathrm{s}^{2}$ is the gravity. The second term on the right hand side of Eq. (2b) accounts for hydrostatic pressure. The pressure field is calculated at each time step following an iterative procedure: (i) the pressure is set to zero at the outermost point of the free surface; (ii) employing the forward integration technique described in Panciroli and Porfiri (2013), the pressure along the outer boundary of the fluid, away from the impacting body, is calculated excluding the body surface, the water free surface and the symmetry boundary; and (iii) using the approach proposed in Baur and Köngeter (1999), the pressure field on the entire fluid bulk is computed. These steps are repeated at each time increment until the pressure field for the whole duration of the impact is reconstructed.

The gradient of the cross-sectional velocity components along the $z$-direction $(\partial u / \partial z$ and $\partial v / \partial z)$ in each crosssectional plane is estimated from the velocity values in that plane and the preceding one, using a backward difference scheme. However, there is a subset of grid points for which the velocity values from the preceding cross-section are not available, being occupied by the cross-section of the model. The shaded area in Figure 5(c) illustrates such overlapped region shared between the fluid at $z=6 \mathrm{~cm}$ and the model at $z=5 \mathrm{~cm}$. To estimate the gradient values in this overlapped region, the available gradient values in the whole fluid bulk at each $z$ are extrapolated using the function "inpaint_nans" in Matlab. This function is based on the inpainting method (Bertalmio et al., 2000), which is used to smoothly interpolate and extrapolate unknown elements in a 2D array.

To elucidate the effect of the axial velocity component on the pressure reconstruction, the pressure field is also calculated by neglecting the axial component, duplicating the approach in Panciroli and Porfiri (2013) for each crosssectional PIV measurement. Note that in this analysis, we still retain the entire cross-sectional PIV dataset, rather than considering only the flow on the mid-span of the model as in Panciroli and Porfiri (2013); Shams et al. (2015); Panciroli et al. (2015).

\section{Results and discussion}

\subsection{Pile-up analysis}

To quantify the pile-up phenomenon, we calculate the so-called pile up coefficient $\phi=c / c^{\star}$ for each crosssectional plane. This quantity is defined as the ratio between the wetted width $c$, defined from the location of the root of the water jet, and the corresponding reference width $c^{\star}$, see Fig. 5. Figure 6 displays the pile-up coefficient as a function of the entry depth for all the cross-sectional PIV locations, presented for the first repetition. In agreement with results presented by Panciroli et al. (2015) on the impact of wedge-like models with a square base and varying geometric curvature, the pile-up coefficient is lower than $\pi / 2$. This suggests that the original prediction of $\pi / 2$ by Wagner (1932) could be used as an empirical upper bound for the pile-up coefficient for both 2D and 3D impacts. Further evidence for this hypothesis can be found in Facci et al. (2015) and Mei et al. (1999), where theoretical and numerical schemes are proposed for the analysis of water entry problems. For each cross-section, the pile-up coefficient shows a similar behavior, whereby it starts from 1 and gradually increases to approximately 1.25 as the model enters the water surface everywhere except of the ends, where it reaches a smaller value of 1.1.

The observed range of variation of the pile-up coefficient is slightly different from the interval between 1.1 and 1.4 reported by Panciroli et al. (2015). This difference could be related to 3D effects, which only play a secondary role in the experiments on wedge-like models by Panciroli et al. (2015). To better illustrate the relevance of 3D effects 


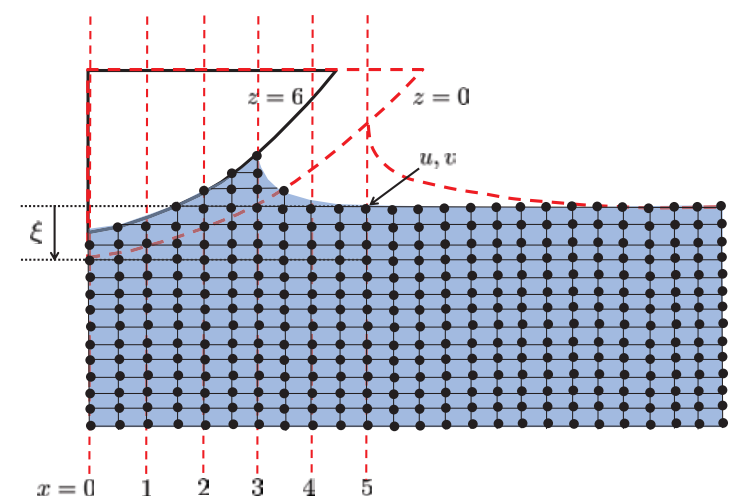

(a)

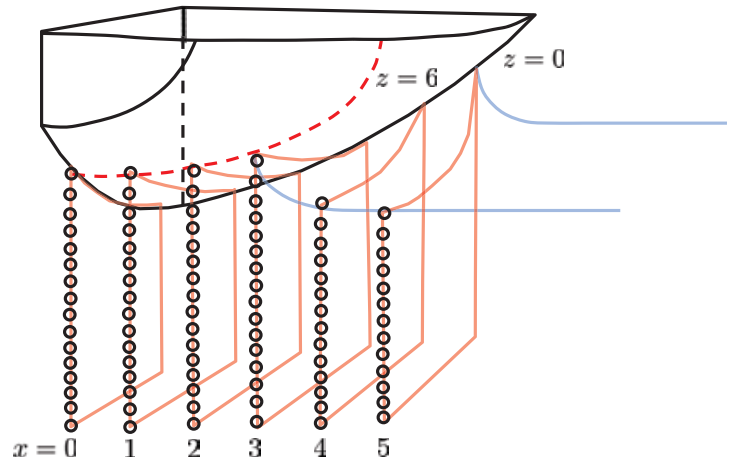

(b)

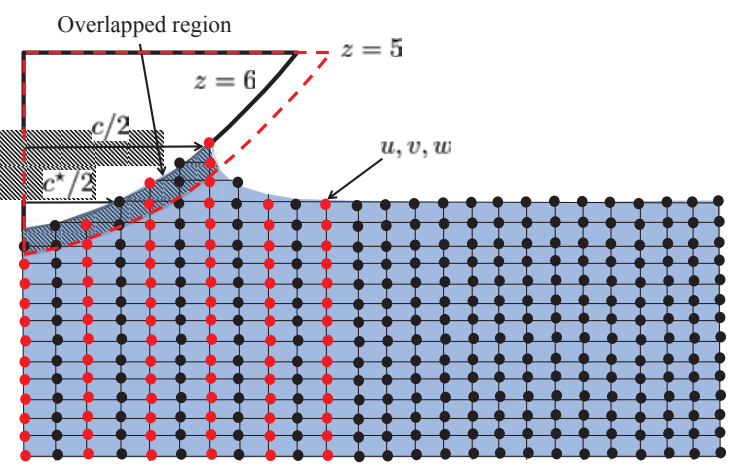

(c)

Figure 5: Interpolation steps (ii), (iii), (iv), and (v); the units of all the numbers is $\mathrm{cm}$. PIV enrichment is illustrated for a representative cross-section at $z=6 \mathrm{~cm}$. Cross-sectional PIV data are enriched with the axial velocity component available from $x=0$ to $5 \mathrm{~cm}$. (a) The velocity grid obtained after PIV analysis for $z=6 \mathrm{~cm}$ is overlaid with the model cross-section and the pile-up shape at $z=0 \mathrm{~cm}$, highlighting the difference between the pile-up shape and the area occupied by the model at each cross-section. (b) Perspective view of the model, illustrating the grid points where the axial velocity component is estimated. (c) The red grid points are enriched with the estimation of the axial velocity. The overlapped region between the velocity grid points at $z=6 \mathrm{~cm}$ and the model cross-section at $z=5 \mathrm{~cm}$, shown as a shaded area, is where the extrapolation of the velocity gradients $\partial u / \partial z$ and $\partial v / \partial z$ is performed.

on the water pile-up, we reconstruct the contact line between the model and the fluid, corresponding to the horizontal projection of the last wetted point in each cross-section of the model. By using the time delay data in Fig. 4, in Fig. 7 we combine the time histories of the wetted width for the first repetitions. The contact line is a closed curve which has a rather steep slope with respect to the $z$-axis, different from a purely $2 \mathrm{D}$ solution. Figure 7 displays the experimentally reconstructed contact line for three impact times $t=7.5,12.5$, and $17.5 \mathrm{~ms}$. Based on observations of raw images, it is tenable to hypothesize that the fluid is evacuated in the spray jet at a direction normal to the contact line.

\subsection{Velocity field}

Figure 8 shows cross-sectional PIV images overlaid with contours of the cross-sectional velocity magnitude, at locations $z=0,3,6$, and $9 \mathrm{~cm}$, at $t=17.5 \mathrm{~ms}$ for the first set of repetitions. In agreement with results reported in Jalalisendi et al. (2015), the maximum cross-sectional velocity is attained in the pile-up region and it gradually decreases in the vicinity of the keel. By comparing Figs. 8(a)-(d), we observe that the cross-sectional velocity magnitude decreases away from the mid-span attaining almost a half of the maximum velocity at $z=9 \mathrm{~cm}$. This should be associated with the 3D nature of the flow field, causing an axial flow increasing in intensity away from the mid-span.

Such a scenario is supported by data in Fig. 9, where we display an axial PIV image overlaid with contours of the magnitude of the velocity field in the axial PIV plane at $x=0 \mathrm{~cm}$ for $t=17.5 \mathrm{~ms}$, also presented for the first repetition. The velocity vector is almost vertical at the mid-span. As we approach the end of the model, we note a 

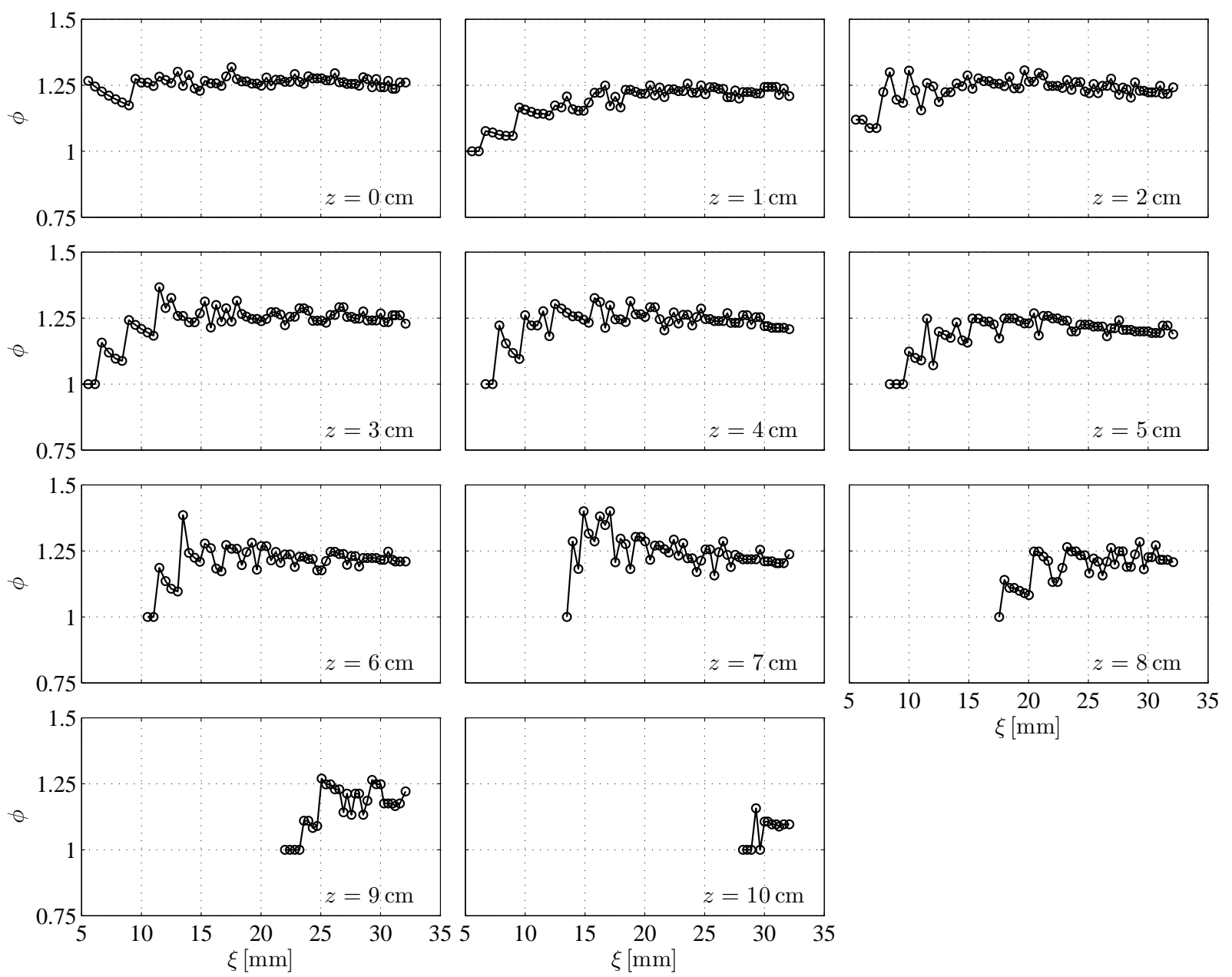

Figure 6: Pile-up coefficients as a function of the entry depth for all the cross-sectional PIV measurement planes. The linear interpolation (solid line) is only presented for ease of illustration. Note that the penetration depth is plotted from $5 \mathrm{~mm}$ so that the pile-up coefficient in the top left panel does not start from 1 , like the other panels.

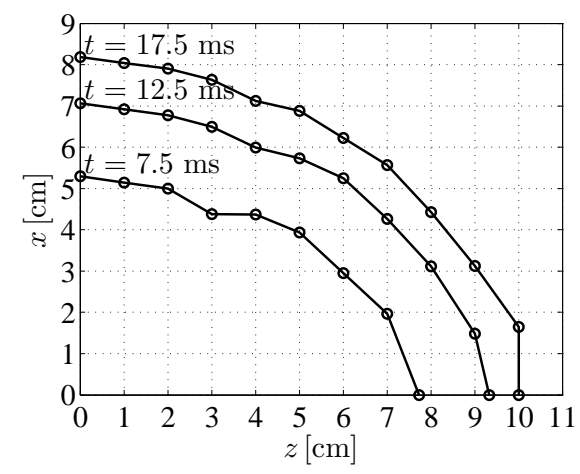

Figure 7: Evolution of the contact line between the fluid and the impacting model over time. The linear interpolation (solid line) is only presented for ease of illustration. 
marked increase of the axial velocity component $w$ along with a robust decrease of $v$. Different from results reported in Jalalisendi et al. (2015), the magnitude of the velocity field in the axial PIV plane is not maximized in the vicinity of the keel, but at half way along the model length.

Similar to Jalalisendi et al. (2015), we also find a cavity on the edges, visible as a bright area in Fig. 9. However, such a cavity is only formed when the water pile-up reaches the edges, in contrast to Jalalisendi et al. (2015) where a cavity is formed in the early stage of the impact. This can be attributed to the geometry considered in Jalalisendi et al. (2015), which makes the flow more prone to separation from the lateral sides that are perpendicular to the keel.

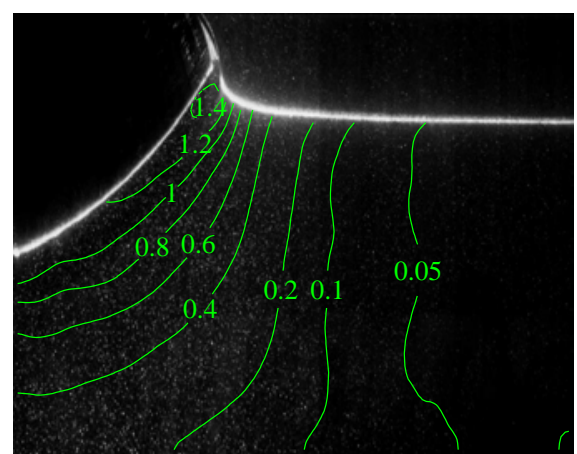

(a)

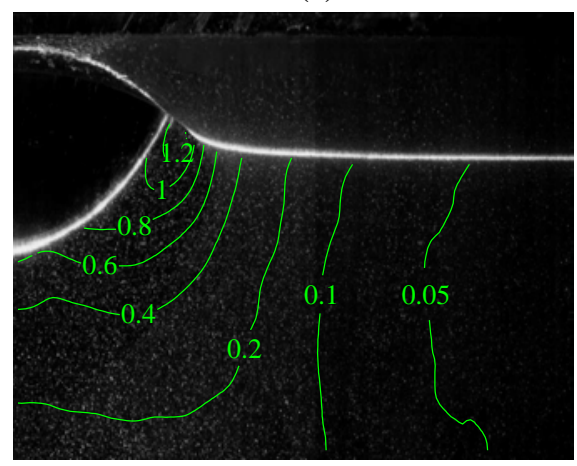

(c)

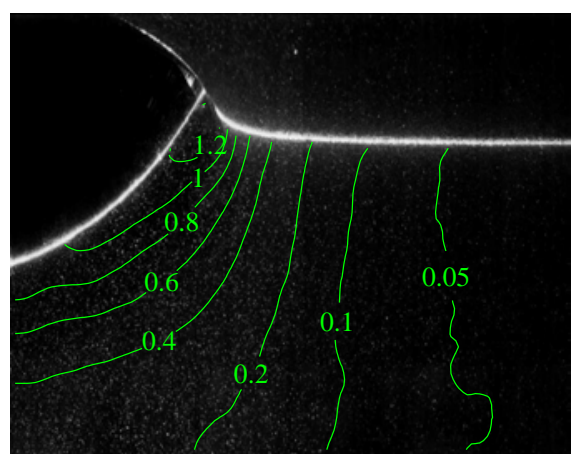

(b)

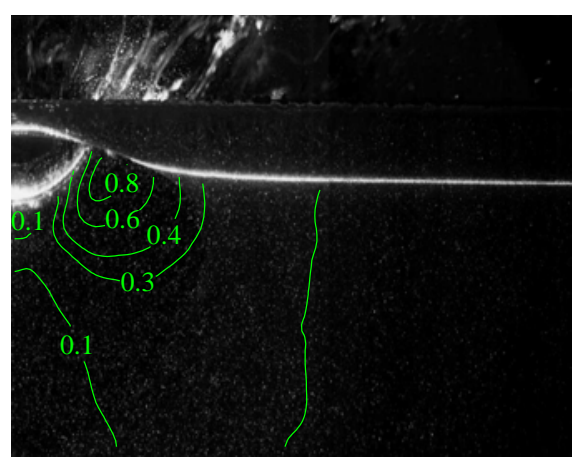

(d)

Figure 8: High speed images with overlaid PIV planar velocity magnitude (computed from $u$ and $v$ ) in $\mathrm{m} / \mathrm{s}$ at $t=17.5 \mathrm{~ms}$ for: (a) $z=0 \mathrm{~cm}$, (b) $z=3 \mathrm{~cm}$, (c) $z=6 \mathrm{~cm}$, and (d) $z=9 \mathrm{~cm}$. The oblate shape of the model is due to the angle of the camera with respect to the optical table. The water splash seen in (c) and (d) is from the prior impact of cross-sections closer to the mid-plane. The horizontal water surface in (c) and (d) is from the front wall of the tank.

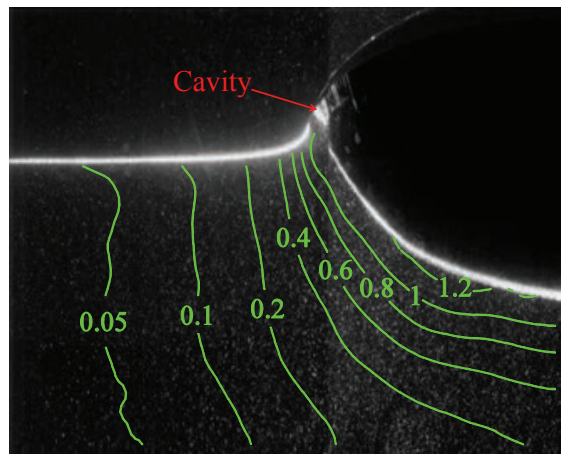

Figure 9: High speed image with overlaid PIV planar velocity magnitude (computed from $v$ and $w$ ) in $\mathrm{m} / \mathrm{s}$ at $x=0 \mathrm{~cm}$ for $t=17.5 \mathrm{~ms}$. 
To elucidate the velocity distribution on the impacting body, in Fig. 10 we display the velocity components $u, v$, and $w$ on the wetted portion of the model as a function of the normalized wetted width, defined as the ratio $X / c$, for the same tests and locations shown in Fig. 8, but for three time instants obtained from the first set of repetitions. In agreement with results reported in Panciroli and Porfiri (2013); Jalalisendi et al. (2015), the cross-sectional velocity components $u$ and $v$ are respectively minimized and maximized in the vicinity of the keel and pile-up, see Figs. 10(a) and (b). The axial velocity component shows a rather different behavior than the cross-sectional components, whereby we find that it is maximized at the keel and minimized in the pile-up, similar to Jalalisendi et al. (2015). Further, we notice that cross-sectional velocity components $u$ and $v$ tend to decrease away from the mid-span, while axial velocity $w$ gently increases toward the ends, different from Jalalisendi et al. (2015).

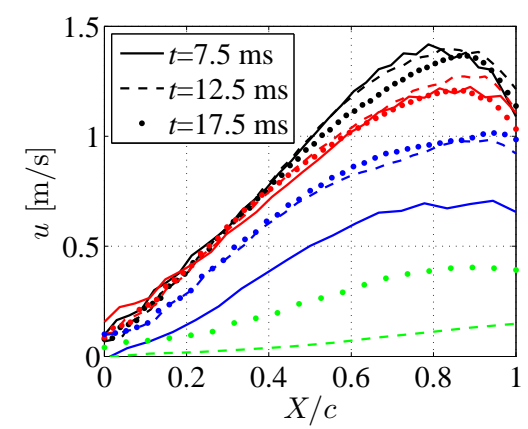

(a)

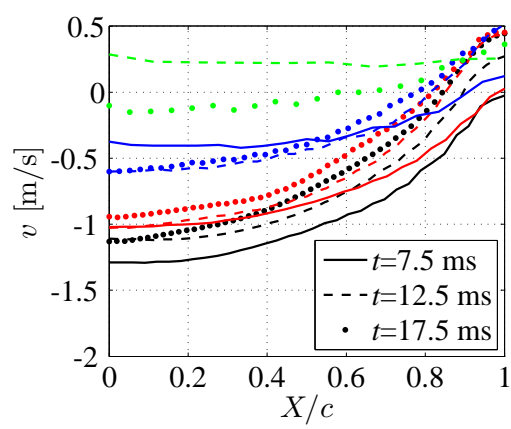

(b)

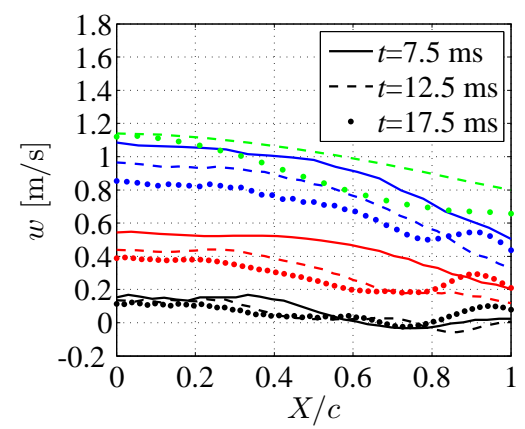

(c)

Figure 10: Velocity components along the model width for three different time instants and four cross-sections: (a) $u$, (b) $v$, and (c) $w$. Black, red, blue, and green lines refer to $z=0,3,6,9 \mathrm{~cm}$, respectively. Note that the model is not wetted at $z=9 \mathrm{~cm}$ for $t=7.5 \mathrm{~ms}$. Note that $c$ is a function of time.

\subsection{Analysis of the energy transferred to the fluid}

Extending the control volume analysis presented in Panciroli et al. (2015) to 3D water impact problems, here we seek to quantify the energy transferred to the risen water, which accounts for the spray jet, where PIV data are not available, and the pile-up. Due to symmetry, we only consider a quarter of the whole fluid domain and divide the regions into volumes $\mathcal{R}_{1}$ and $\mathcal{R}_{2}$, see Fig. 11 (a), where $\mathcal{R}_{1}$ represents the volume occupied by the fluid bulk and $\mathcal{R}_{2}$ represent the volume of the risen water. The rate of change of the energy in $\mathcal{R}_{2}$, which we seek to estimate from the flow measurements in the fluid bulk and the motion of the model, can be written as

$$
\begin{aligned}
& \frac{\mathrm{d}}{\mathrm{d} t} \int_{\mathcal{R}_{2}}\left(\frac{1}{2} \rho\|\mathbf{v}\|^{2}+\rho g y\right) d \mathcal{R}=-\frac{\mathrm{d}}{\mathrm{d} t} \int_{\mathcal{R}_{1}}\left(\frac{1}{2} \rho\|\mathbf{v}\|^{2}+\rho g y\right) d \mathcal{R} \\
& -\int_{\Gamma_{1} \cup \Gamma_{2} \cup \Gamma_{3}}\left(\frac{1}{2}\|\mathbf{v}\|^{2}+g y+\frac{p}{\rho}\right) \rho(\mathbf{v} \cdot \mathbf{n}) d \Gamma-\frac{1}{4}\left(M_{t} \ddot{\xi} \dot{\xi}-M_{t} g \dot{\xi}\right)
\end{aligned}
$$

where $\mathbf{v}$ is the velocity vector in the fluid bulk, $\|\mathbf{v}\|^{2}$ is the velocity magnitude, and $\mathbf{n}$ is the unit normal.

The first integral on the right hand side is the rate of change of the energy in the fluid bulk and is computed from the cross-sectional and axial velocity data. Specifically, for each $z$ value, we calculate the energy per unit length transferred to the fluid bulk using the control surface $\Gamma$, see Fig. 11(b), for each repetition. Then, we average across repetitions and integrate along the length using a trapezoidal rule to obtain the total energy transferred to the fluid bulk. Notably, for cross-sectional planes in which the model is still not present, the estimation of the energy is only based on the axial PIV dataset. Finally, the time history of the energy imparted to the fluid bulk is numerically differentiated in time, using the second order finite difference scheme. A similar procedure is used to calculate the integrals on the surfaces $\Gamma_{1}, \Gamma_{2}$, and $\Gamma_{3}$, which are associated with the energy flow outside the control volume. Therein, the pressure field is obtained using the pressure integration technique described in Section 3.2. Finally, the acceleration and velocity of the model measured in the separate set of experiments are used to evaluate the rate of change of the energy of the falling model corresponding to the last summand on the right hand side of Eq. (3). Note that this term 
also defines the rate of change of the energy transferred to the whole fluid. Equation (3) is integrated in time using the cumulative trapezoidal method to reconstruct the full energy budget of the impact.

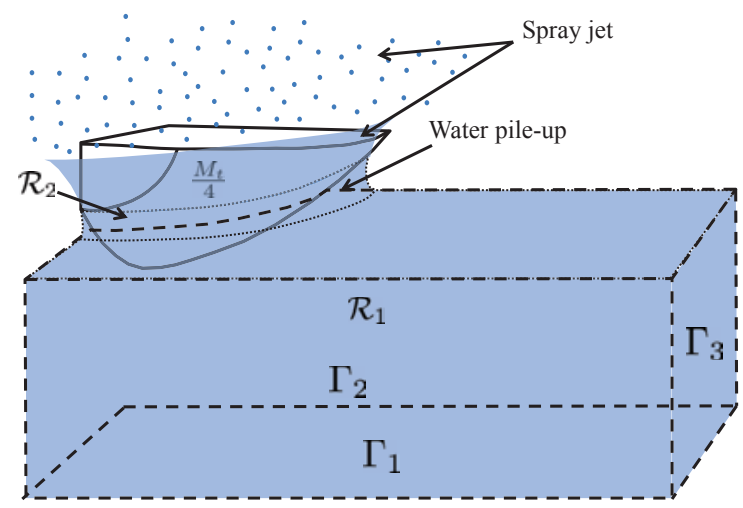

(a)

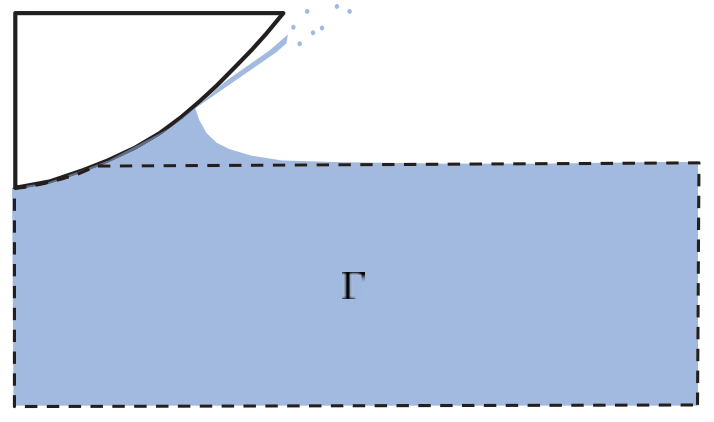

(b)

Figure 11: (a) Schematic of the control volume analysis with overlaid notations. (b) Schematic of the control surface for each cross-sectional PIV experiment.

Figure 12 shows the total energy transferred to the whole fluid along with the energy imparted to the risen water, presented both as a fraction of the model initial energy $1 / 2 M_{t} \dot{\xi}^{2}(0)$. The total energy transferred to the whole fluid is computed from the variation of the energy of the model for the duration of the impact. For completeness, we calculate the energy imparted to the risen water by using the full 3D velocity field or the simpler $2 \mathrm{D}$ estimation without local enrichment. Experimental results demonstrate that almost all the energy transferred to the fluid is imparted to the pileup and the spray jet and that the 3D effects are almost negligible for $\xi \leq 15 \mathrm{~mm}$. These predictions are in agreement with results reported in Panciroli et al. (2015), where it is suggested that 3D effects should have a secondary role during the early stage of the impact. For $\xi \geq 15 \mathrm{~mm}$, 3D effects become more relevant, and at the end of the impact they contribute to approximately $5 \%$ of the total energy imparted to the fluid. Figure 12 also suggests that the energy transferred to the fluid bulk increases as the model penetrates the water surface, whereby we observe a larger discrepancy between the total energy transferred to the fluid and the energy imparted to the risen water. These findings are in line with the analysis of Scolan and Korobkin (2003), which predicts that the main portion of the impact energy is transmitted to the pile-up and spray jet for a decelerating body.

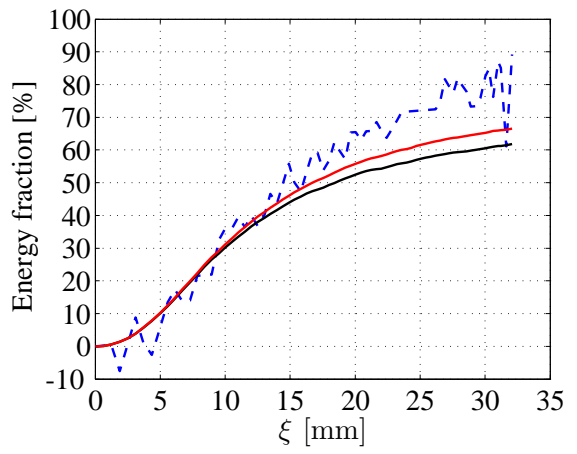

Figure 12: Energy transferred to the whole fluid and portion imparted to the risen water, both calculated as a percentage of the model initial energy $1 / 2 M_{t} \dot{\xi}^{2}(0)$. The dashed blue line is the energy imparted to the whole fluid, computed from the change of the energy of the falling model during the impact. Black and red lines refer to the energy imparted to the water pile-up and spray jet using the estimation of the axial velocity component (3D) or not (2D), respectively; note that the $2 \mathrm{D}$ computation overestimates the $3 \mathrm{D}$ prediction. 

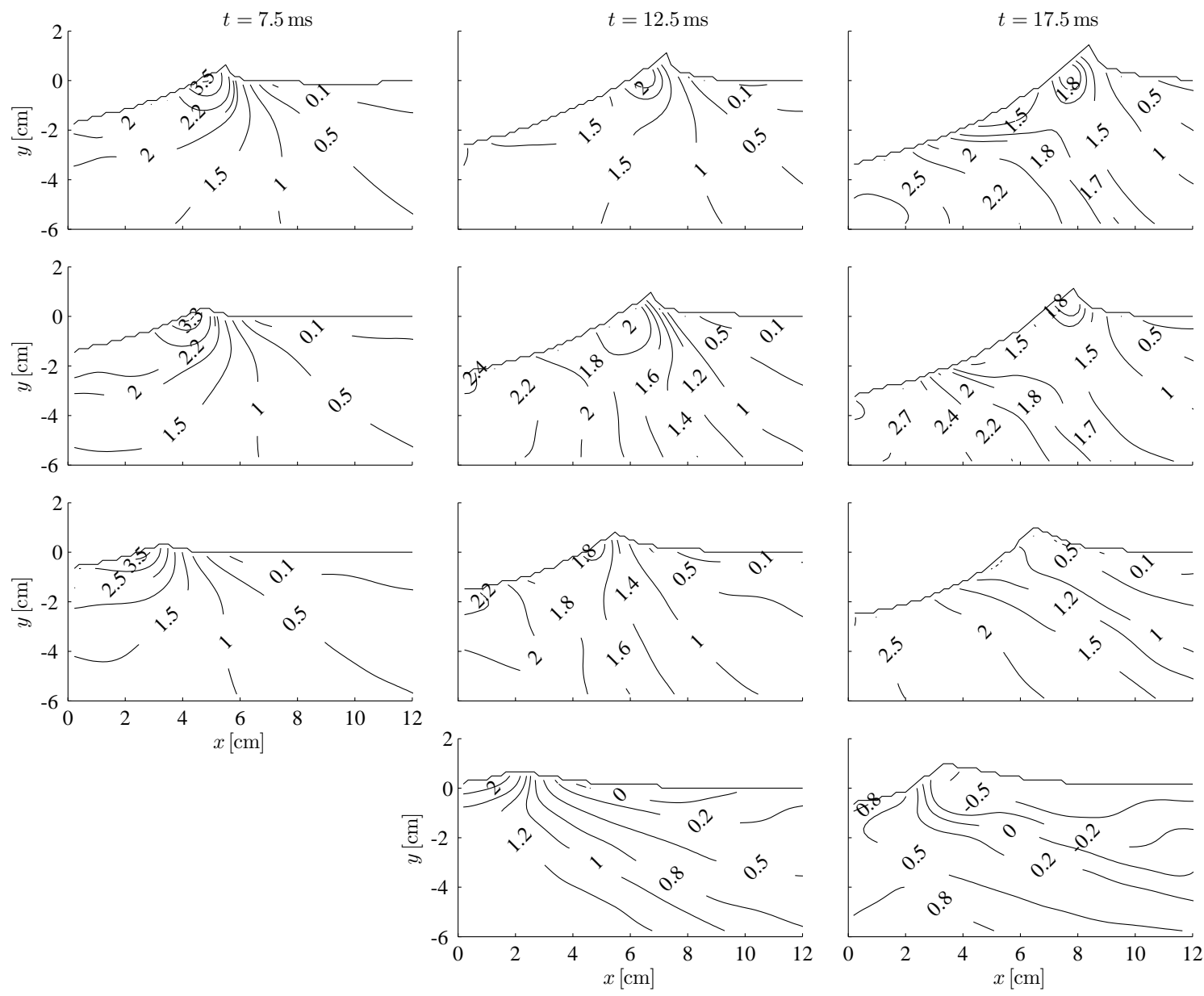

Figure 13: Normalized pressure distribution at $z=0 \mathrm{~cm}$ (first row), $z=3 \mathrm{~cm}$ (second row), $z=6 \mathrm{~cm}$ (third row), and $z=9 \mathrm{~cm}$ (fourth row) for three different time instants $t=7.5,12.5$, and $17.5 \mathrm{~ms}$. Note that the model is not wetted at $z=9 \mathrm{~cm}$ for $t=7.5 \mathrm{~ms}$.

\subsection{Reconstructed pressure field}

Here, we present the normalized pressure field reconstructed using the method detailed in Sec. 3.2. Figure 13 displays the pressure fields for the same tests shown in Fig. 10; the pressure values are normalized by $1 / 2 \rho \dot{\xi}^{2}(t)$.

In agreement with results in Jalalisendi et al. (2015); Panciroli and Porfiri (2013), in the initial stage of impact, $t=7.5 \mathrm{~ms}$, the pressure is maximized in the pile-up and minimized at the keel for all the measurement locations. As the model further penetrates the water surface, $t=12.5$ and $17.5 \mathrm{~ms}$, the pressure at the keel reaches values as large as those attained in the pile-up. Different than Jalalisendi et al. (2015), we do not observe a consistent decrease in the pressure distribution away from the mid-span of the model. This difference may be ascribed to the curvature of the model in the $Y Z$-plane, which causes the keel to be wetted at different time instants during the water entry. Thus, the flow on each wetted cross-section evolves from a well developed flow structure, which is likely to induce a more modest variation of the pressure distribution along the length of the model. Note that light reflections at the water free surface cause uncertainties in the PIV measurements resulting into non-zero pressure values at the free surface (Panciroli and Porfiri, 2013).

During the later state of the impact, negative pressure values are observed at $z=9 \mathrm{~cm}$ for $t=17.5 \mathrm{~ms}$, while the pressure is consistently positive during the whole duration of the impact for any other cross-sections. Similar findings 
are reported in Jalalisendi et al. (2015), where pressure levels less than atmospheric pressure are seen as the model decelerates during water entry. As also explained in Korobkin (2003), for a free falling body in which the velocity decreases during the impact, the pressure might attain values less than the atmospheric pressure values.

\subsection{Hydrodynamic loading}

The hydrodynamic loading on the surface of the model is displayed in Fig. 14 for the first set of repetitions. Results are shown for three time instants $t=7.5,12.5$, and $17.5 \mathrm{~ms}$. These findings shed light on the temporal and spatial evolution of the hydrodynamic loading experienced by the model during impact. Initially, the loading is maximized in the region close to the contact line and minimized close to the keel near the mid-span. This loading distribution changes as the model penetrates the water surface, whereby we observe a rather flat distribution with slightly larger values attained close to the keel.
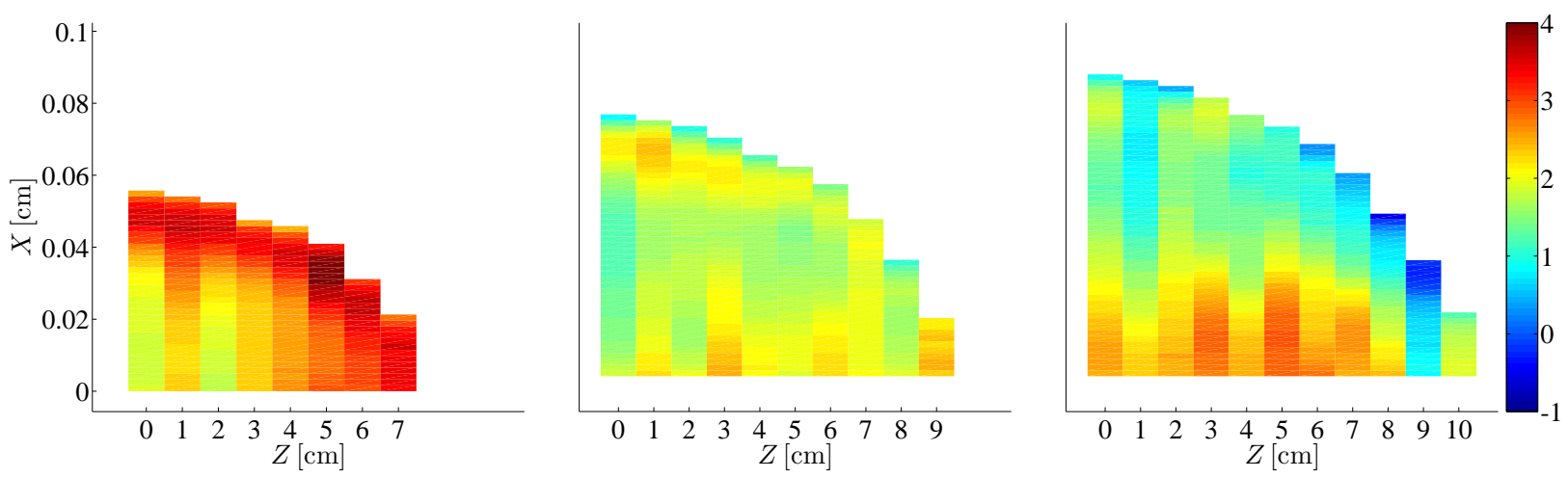

Figure 14: Normalized pressure distribution on the surface of the model at three time instants: (a) $t=7.5 \mathrm{~ms}$, (b) $t=12.5 \mathrm{~ms}$, and (c) $t=17.5 \mathrm{~ms}$.

To further elucidate the hydrodynamic loading on the structure, we display the normalized pressure profile on the normalized wetted width of the model at selected cross-sectional PIV locations in Fig. 15. The results are presented for the same tests shown in Fig. 13. These findings confirm that the pressure is maximized in the pile-up region during the earlier stage of the impact and minimized at the keel. This distribution radically changes as the model penetrates the water surface, whereby we observe that the pressure at the keel reaches values as large as those in the pile-up. Thus, the location of the minimum normalized pressure shifts towards values close to 0.5 rather than 0 . We also display the hydrodynamic loading for the same tests by neglecting the axial velocity component. Results indicate that the hydrodynamic loading is almost unaffected by the axial velocity near the mid-span where the flow is largely 2D. Moving from the mid-span to the ends, the effect of the axial velocity on the hydrodynamic loading gradually increases, causing the reconstruction based on 2D data to overestimate the hydroynamic loading.

Figure 16 displays the maximum and minimum value of the hydrodynamic loading on the wetted model as a function of the entry depth for the same tests presented in Fig. 15 . Here, the results are normalized by $1 / 2 \rho \dot{\xi}^{2}(0)$ to better illustrate the pressure variations as the entry depth increases. Results show that the hydrodynamic loading decrease during water entry, as the model decelerates. However, we find that the dependence of the loading with respect to the penetration depth is less evident for locations close to the ends, whereby we observe that for $z=9 \mathrm{~cm}$ the pressure is almost insensitive to changes in $\xi$. Specifically, at location $z=9 \mathrm{~cm}$ the graph remains almost flat. For completeness, we also report the hydrodynamic loading computed by neglecting the axial velocity component, which indicates that the axial velocity component has a significant role in shaping the hydrodynamic loading close to the ends and especially in the later stage of the impact.

The total force acting on the model at any time instant can be calculated by integrating the pressure value on the wetted surface. Specifically, we utilize the following formula:

$$
F(t)=\int_{\mathcal{S}} \hat{p}(X, Z, t)\left(\mathbf{n}_{s}(X, Y) \cdot j\right) d \mathcal{S}=\int_{\mathcal{S}} \hat{p}(X, Z, t) / \sqrt{\left(\frac{\partial Y(X, Z)}{\partial X}\right)^{2}+\left(\frac{\partial Y(X, Z)}{\partial Z}\right)^{2}+1} d \mathcal{S}
$$



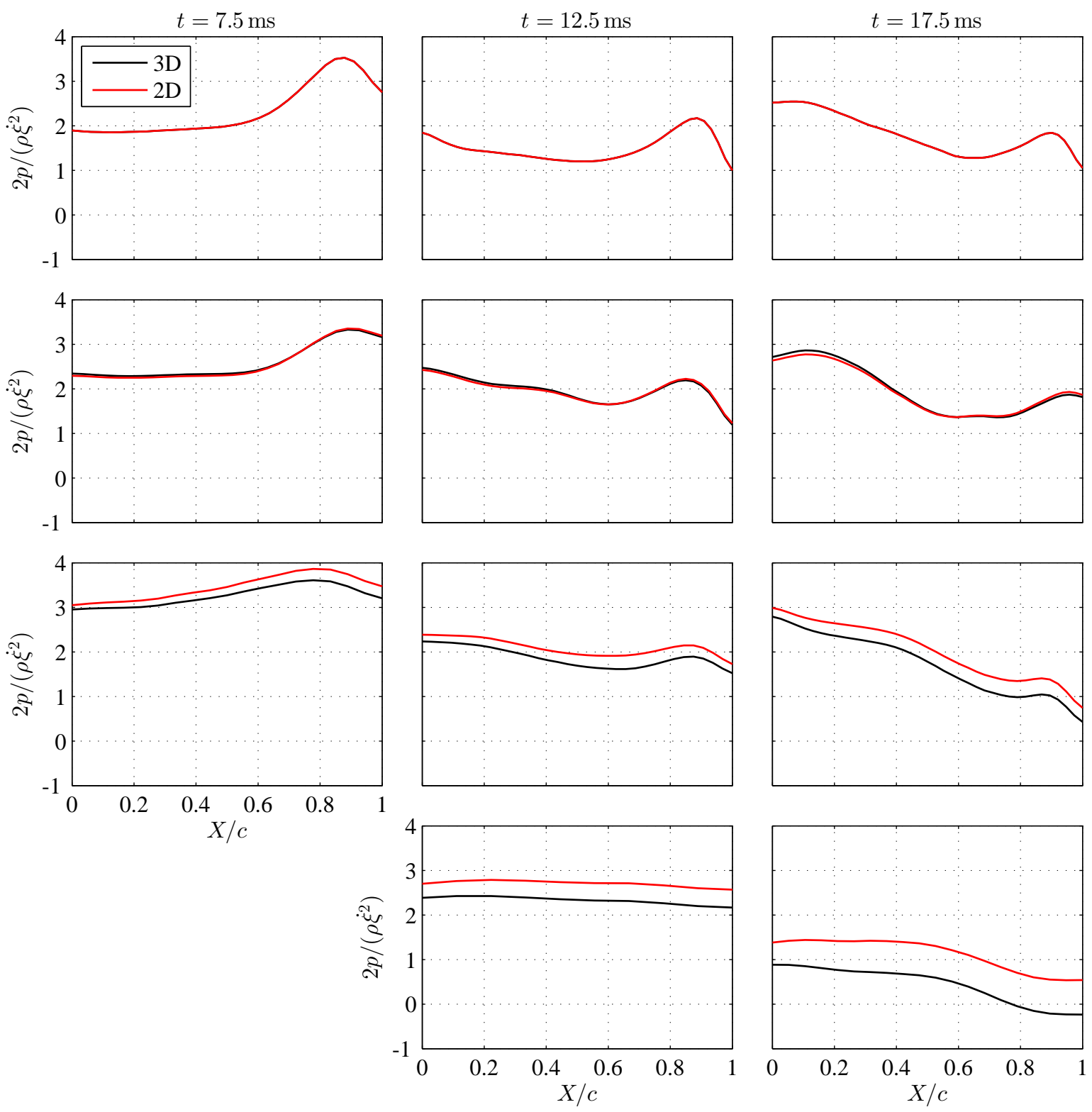

Figure 15: Normalized hydrodynamic loading on the wetted part of model at $z=0 \mathrm{~cm}$ (first row), $z=3 \mathrm{~cm}$ (second row), $z=6 \mathrm{~cm}$ (third row), and $z=9 \mathrm{~cm}$ (fourth row) for three different time instants $t=7.5,12.5$, and $17.5 \mathrm{~ms}$. Note that the model is not wetted at $z=9 \mathrm{~cm}$ for $t=7.5 \mathrm{~ms}$. Black and red lines refer to the reconstruction of the hydrodynamic loading using the estimation of the axial velocity component (3D) or not (2D), respectively. Note that $\dot{\xi}$ and $c$ are functions of time.

Here, $\hat{p}(X, Z, t)$ is the pressure on the model surface at location $(X, Y(X, Z), Z)$ and time $\mathbf{t} ; \mathbf{n}_{s}(X, Z)$ and $j$ are the unit normal to the model at the location $(X, Y(X, Z), Z)$ and the unit normal in $Y$-direction, respectively; and $\mathcal{S}$ is the wetted surface of the model at time $t$. The integral in Eq. (4) is calculated numerically using a trapezoidal rule.

The force per unit length at each cross-sectional PIV plane can be calculated by fixing the value of $Z$ and restricting the integral in Eq. (4) to the cross-section of $\mathcal{S}$. Figure 17 displays the time histories of the force per unit length for the same tests presented in Fig. 16. As expected from the analysis of the hydrodynamic loading in Fig. 15, the magnitude of the force per unit length decreases from the mid-span to the ends of the model. The severity of such a reduction 


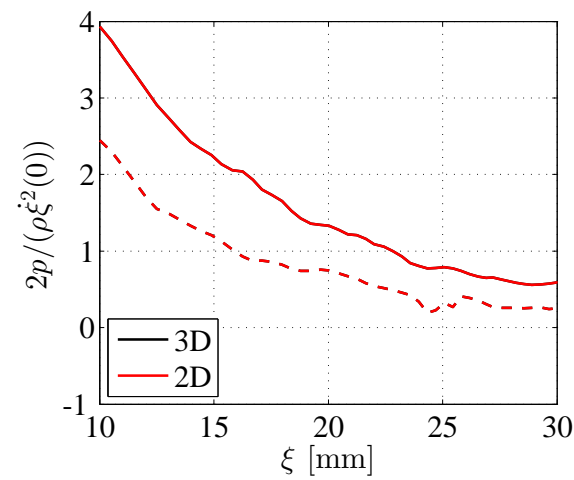

(a)

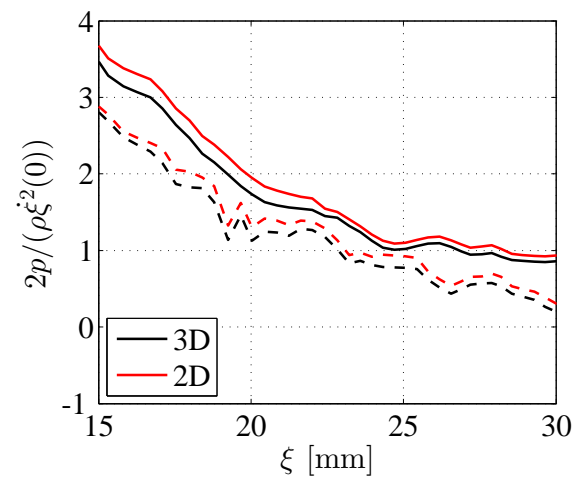

(c)

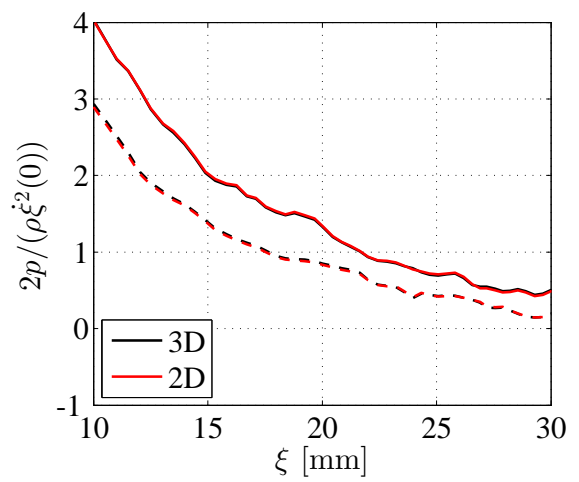

(b)

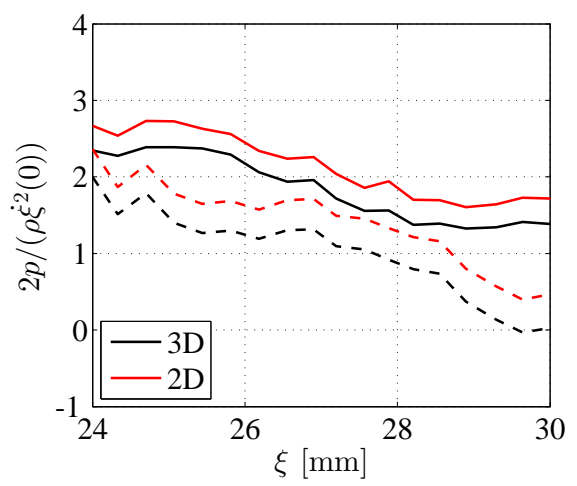

(d)

Figure 16: Maximum (solid) and minimum (dashed) values of the normalized hydrodynamic loading on the wetted part of the model versus the entry depth for multiple locations: (a) $z=0 \mathrm{~cm}$, (b) $z=3 \mathrm{~cm}$, (c) $z=6 \mathrm{~cm}$, and (d) $z=9 \mathrm{~cm}$. Black and red lines refer to results using the estimation of the axial velocity component (3D) or not (2D), respectively.

depends nonlinearly on the $z$-coordinate, whereby we observe a rather modest change by varying $z$ from 0 to $3 \mathrm{~cm}$ in contrast to a fivefold reduction by changing $z$ from 6 to $9 \mathrm{~cm}$. Notably, the force per unit length is maximized at a different time for each cross-section. As the force per unit length at a cross-section starts decreasing, the neighboring cross-section experiences a sudden raise in its loading. These findings confirm that the role of the axial velocity is only relevant away from the mid-span of the model, resulting into noticeable overpredictions of the hydrodynamic loading from $2 \mathrm{D}$ data in the vicinity of the ends.

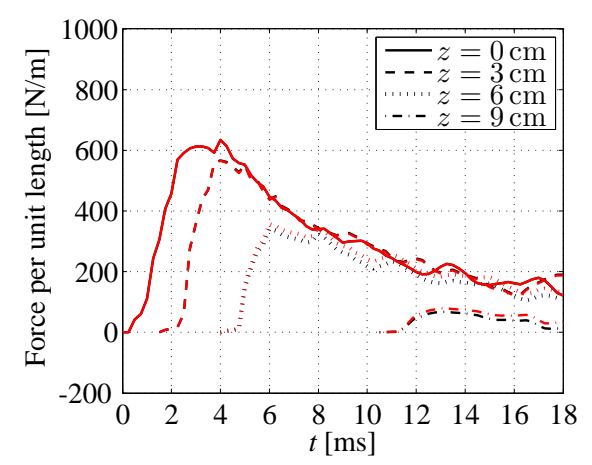

Figure 17: Force per unit length on the wetted part of the model at multiple locations. Black and red colors refer to results using the estimation of the axial velocity component (3D) or not (2D), respectively. 
To further illustrate the dependence of the force per unit length on the $z$-coordinate, in Fig. 18 we present the averaged values of the maximum force per unit length across the three repetitions for all the 11 cross-sections. For completeness, we also report the corresponding values calculated by neglecting the axial velocity component. Our results indicate that the peak force per unit length is nearly constant for $0 \leq z \leq 3 \mathrm{~cm}$, while gradually decreasing for $z \geq 3 \mathrm{~cm}$ so that the end of the model experiences a far more modest loading than the cross-section at the mid-span. By fitting the force per unit length reconstructed using the 3D enriched dataset by a third order polynomial, we find a correlation coefficient equal of 0.995 , which suggests a highly nonlinear behavior. The axial velocity component has a moderate effect on the maximum force per unit length, with the maximum difference between $2 \mathrm{D}$ and 3D values being equal to $16.3 \%$.

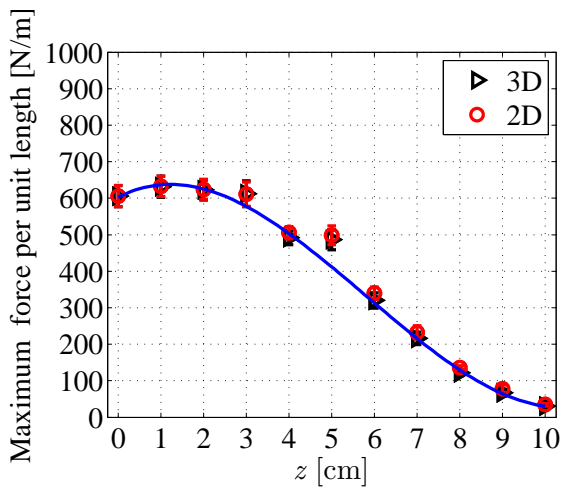

Figure 18: Maximum force per unit length for all the measurement locations along the model length. Black triangles and red circles refer to average values of the three repetitions using the estimation of the axial velocity component (3D) or not (2D), respectively. Error bars indicate the maximum difference between the repetitions and the average. The blue line is a third order polynomial fit of the averaged $3 \mathrm{D}$ data $\left(1.59 z^{3}-27.72 z^{2}+61.03 z+\right.$ 600.10 where $z$ is in $\mathrm{cm}$ ).

To offer some independent validation of PIV results, we compare the reconstructed total force exerted on the impacting model in Eq. (4) with data from the accelerometer, see Fig. 19. Both the accelerometer and PIV predictions are averaged across three trials, and PIV predictions are calculated twice by considering (3D) or neglecting (2D) the axial velocity component. The comparison confirms the accuracy of PIV-based pressure reconstruction, especially after the very first stage of the impact, $t>6 \mathrm{~ms}$. The discrepancy between PIV and accelerometer data should be ascribed to the underestimation of the velocity field during the early stage of the impact. As also discussed in Panciroli and Porfiri (2013); Nila et al. (2013); Jalalisendi et al. (2015); Facci et al. (2015), the underestimation of the fluid velocity is likely due to the large velocity gradients in the proximity of the impacting model, that result into large variations of the velocity field within the interrogation windows which are only averaged by PIV. In agreement with results in Fig. 17, we find that the axial velocity component has a rather modest effect on the total force experienced by the model. In fact, while the normalized hydrodynamic loading close to the end of the model is dramatically affected by the axial component of the velocity, see Fig. 15, its contribution on the total force is only marginal.

However, this does not imply that a single planar PIV measurement would be sufficient to accurately predict the total force as found in Panciroli and Porfiri (2013) for a wedge-like model. By multiplying the force per unit length at the mid-span in Fig. 17 by the model length, we find a total force that is $59.1 \%$ larger than the total force reconstructed using the pressure reconstruction methodology reported in Fig. 19 (computed as the normalized root mean square error).

\section{Conclusions}

In this paper, we have studied the water entry of a solid body into a quiescent fluid through PIV. The body was designed to model a ship hull, with a concave cross-section whose curvature changes along the length. The model was $20 \mathrm{~cm}$ long, $16 \mathrm{~cm}$ wide, and $5.6 \mathrm{~cm}$ tall, and weighted $0.601 \mathrm{~kg}$. The model entered the water surface with pure vertical velocity, falling from a height of $50 \mathrm{~cm}$ to attain an entry speed of $2.47 \mathrm{~m} / \mathrm{s}$. The PIV technique proposed in Jalalisendi et al. (2015) was extended to measure the 3D velocity field of this complex model during water entry. PIV experiments 


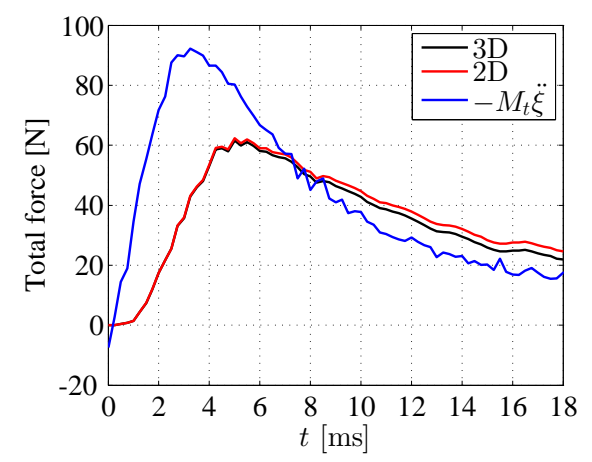

Figure 19: Comparison between the total force acting on the model computed from the measured acceleration and PIV-based pressure reconstruction estimation. The blue line is the accelerometer data $\left(-M_{t} \ddot{\xi}(t)\right)$, while the black and red lines refer to results for pressure reconstruction using the estimation of axial velocity (3D) or not (2D), respectively.

were conducted on 11 cross-sections along the length of the model and 9 cross-sections along the width to accurately scan the fluid domain and resolve the velocity field in the fluid bulk and pile-up region. The pressure field in the fluid was then reconstructed through the integration of the incompressible 3D Navier-Stokes equations, accounting for the evolution of both the free-surface and the fluid-solid interface. Through a control volume analysis, we computed the energy transferred to the fluid, dissecting the contribution to the risen water. By evaluating the pressure on the wetted surface of the model, we estimated the hydrodynamic loading during water entry as a function of time and position on the model.

Our results indicate that the formation of the water pile-up is influenced by $3 \mathrm{D}$ phenomena, whereby we observe a rather smaller range of variation of the pile-up coefficient in comparison to 2D geometries (Panciroli et al., 2015). The intensity of the cross-sectional velocity is maximized at the mid-span of the model, and gently decreases toward the edges, where the axial velocity component is stronger. As time advances, the model decelerates and the energy imparted to the fluid increases. We find that almost all the energy is transmitted to the risen water in the early stage of the impact, and as the model further penetrates the water surface we observe an increase in the energy contribution to the fluid bulk. Notably, neglecting the axial velocity component results into a modest overprediction of the energy transmitted to the risen water.

The 3D nature of the velocity field translates into a robust variation of the pressure field in the fluid domain, with smaller pressure values generally attained away from the cross-section at the mid-span of the model and values lower than the atmospheric pressure in the later stage of the impact. As a result, the hydrodynamic loading experienced by the model exhibits a complex dependence on time and the position on the wetted surface. In the early stage of the impact, the hydrodynamic loading is maximized in the pile-up region. As the model further penetrates the water surface, the hydrodynamic loading increases in the vicinity of the keel reaching values even larger than those attained close to the pile-up. The hydrodynamic loading significantly varies with respect to the length of the model due to both the increase in the water surface and the deceleration of the model during impact. Close to the ends, we find that the hydrodynamic loading is relatively flat with respect to the coordinate along the width. Therein, the axial velocity component has a significant role in shaping the hydrodynamic loading, noted by comparing findings obtained by neglecting the axial velocity component in the pressure reconstruction. In addition, the maximum force per unit length nonlinearly depends on the coordinate along the length, with considerably larger values attained in the vicinity of the mid-span. Thus, estimating the force experienced by the model using only planar PIV data at a select cross-section would lead to rather poor estimations.

The results of our work are expected to aid in the validation of mathematical models of 3D water entry problems, which require a detailed resolution of both the hydrodynamic loading and the flow physics. In addition, our findings can inform the design of new experiments using planar PIV and offer insight to researchers and practitioners toward assessing the role of $3 \mathrm{D}$ phenomena for complex geometries. 


\section{Acknowledgment}

This work has been supported by the Office of Naval Research Grant N00014-10-1-0988 with Dr. Y.D.S. Rajapakse as the program manager. Views expressed herein are those of the authors and not of the funding agencies. The authors are grateful to Mr. Adel Shams for his help with the design of the model and the experiments. 


\section{References}

Abrate, S., 2013. Hull slamming. Applied Mechanics Reviews 64, 060803.

Battistin, D., Iafrati, A., 2004. A numerical model for the jet flow generated by water impact. Journal of Engineering Mathematics 48 (3-4), 353-374.

Battley, M., Allen, T., 2012. Servo-hydraulic system for controlled velocity water impact of marine sandwich panels. Experimental Mechanics $52(1), 95-106$

Baur, T., Köngeter, J., 1999. PIV with high temporal resolution for the determination of local pressure reductions from coherent turbulence phenomena. In: 3rd International Workshop on PIV'99, Santa Barbara, CA, USA. pp. 101-106.

Bertalmio, M., Sapiro, G., Caselles, V., Ballester, C., 2000. Image inpainting. In: Proceedings of the 27th annual conference on Computer graphics and interactive techniques, New Orleans, LA, USA. ACM Press/Addison-Wesley Publishing Co., pp. 417-424.

Chuang, S. L., 1966. Slamming of rigid wedge-shaped bodies with various deadrise angles. Technical Report DTMB-2268.

El Malki Alaoui, A., Nême, A., Scolan, Y. M., 2015. Experimental investigation of hydrodynamic loads and pressure distribution during a pyramid water entry. Journal of Fluids and Structures 54, 925-935.

El Malki Alaoui, A., Nême, A., Tassin, A., Jacques, N., 2012. Experimental study of coefficients during vertical water entry of axisymmetric rigid shapes at constant speeds. Applied Ocean Research 37, 183-197.

Facci, A. L., Panciroli, R., Ubertini, S., Porfiri, M., 2015. Assessment of PIV-based analysis of water entry problems through synthetic numerical datasets. Journal of Fluids and Structures 55, 484-500.

Faltinsen, O. M., 1990. Sea Loads on Ships and Offshore Structures. Cambridge University Press, New York, NY.

Faltinsen, O. M., 1997. The effect of hydroelasticity on ship slamming. Philosophical Transactions of the Royal Society of London. Series A: Mathematical, Physical and Engineering Sciences 355 (1724), 575-591.

Faltinsen, O. M., 2006. Hydrodynamics of High-Speed Marine Vehicles. Cambridge University Press, New York, NY.

Faltinsen, O. M., Landrini, M., Greco, M., 2004. Slamming in marine applications. Journal of Engineering Mathematics 48 (3-4), 187-217.

Garcia, D., 2010. Robust smoothing of gridded data in one and higher dimensions with missing values. Computational Statistics and Data Analysis 54 (4), 1167-1178.

Gazzola, T., Korobkin, A. A., Malenica, S., Scolan, Y. M., 2005. Three-dimensional Wagner problem using variational inequalities. In: Proceedings of the International 20th Workshop on Water Waves and Floating Bodies, Longyearbyen, Norway, May.

Gu, X., Moan, T., 2002. Long-term fatigue damage of ship structures under nonlinear wave loads. Marine Technology 39 (2), $95-104$.

Heikkila, J., Silvén, O., 1997. A four-step camera calibration procedure with implicit image correction. In: IEEE Computer Society Conference on Computer Vision and Pattern Recognition, San Juan, PR. pp. 1106-1112.

Hughes, K., Vignjevic, R., Campbell, J., De Vuyst, T., Djordjevic, N., Papagiannis, L., 2013. From aerospace to offshore: Bridging the numerical simulation gaps-simulation advancements for fluid structure interaction problems. International Journal of Impact Engineering 61, 48-63.

Jalalisendi, M., Shams, A., Panciroli, R., Porfiri, M., 2015. Experimental reconstruction of three-dimensional hydrodynamic loading in water entry problems through particle image velocimetry. Experiments in Fluids 56 (2), 1-17.

Judge, C., Troesch, A., Perlin, M., 2004. Initial water impact of a wedge at vertical and oblique angles. Journal of Engineering Mathematics 48 (3-4), 279-303.

Khabakhpasheva, T. I., Korobkin, A. A., 2013. Elastic wedge impact onto a liquid surface: Wagner's solution and approximate models. Journal of Fluids and Structures 36, 32-49.

Korobkin, A. A., 2002. The entry of an elliptical paraboloid into a liquid at variable velocity. Journal of Applied Mathematics and Mechanics $66(1), 39-48$

Korobkin, A. A., 2003. cavitation in liquid impact problems. In: Fifth International Symposium on Cavitation, Osaka, Japan.

Korobkin, A. A., 2004. Analytical models of water impact. European Journal of Applied Mathematics 15 (6), 821-838.

Korobkin, A. A., 2005. Three-dimensional nonlinear theory of water impact. In: Proceedings 18th International Congress of Mechanical Engineering (COBEM), Ouro Preto, Minas Gerais, Brazil.

Korobkin, A. A., Pukhnachov, V. V., 1988. Initial stage of water impact. Annual Review of Fluid Mechanics 20 (1), $159-185$.

Lin, H. J., Perlin, M., 1998. Improved methods for thin, surface boundary layer investigations. Experiments in Fluids 25 (5-6), 431-444.

Mei, X., Liu, Y., Yue, D. K., 1999. On the water impact of general two-dimensional sections. Applied Ocean Research 21 (1), 1-15.

Nila, A., Vanlanduit, S., Vepa, S., Van Paepegem, W., 2013. A PIV-based method for estimating slamming loads during water entry of rigid bodies. Measurement Science and Technology 24 (4), 045303.

Nobach, H., Damaschke, N., Tropea, C., 2005. High-precision sub-pixel interpolation in particle image velocimetry image processing. Experiments in Fluids 39 (2), 299-304

Panciroli, R., Porfiri, M., 2013. Evaluation of the pressure field on a rigid body entering a quiescent fluid through particle image velocimetry. Experiments in Fluids 54 (12), 1630

Panciroli, R., Porfiri, M., 2015. Analysis of hydroelastic slamming through particle image velocimetry. Journal of Sound and Vibration 347, 63-78.

Panciroli, R., Shams, A., Porfiri, M., 2015. Experiments on the water entry of curved wedges: High speed imaging and particle image velocimetry. Ocean Engineering 94, 213-222.

Panton, R. L., 1994. Incompressible Flow. John Wiley \& Sons, New York, NY.

Qin, Z., Batra, R. C., 2009. Local slamming impact of sandwich composite hulls. International Journal of Solids and Structures 46 (10), $2011-2035$.

Raffel, M., Willert, C., Wereley, S., Kompenhans, J., 2007. Particle Image Velocimetry: A Practical Guide. Springer, New York.

Scarano, F., Riethmuller, M., 1999. Iterative multigrid approach in PIV image processing with discrete window offset. Experiments in Fluids 26 (6), 513-523.

Scarano, F., Riethmuller, M. L., 2000. Advances in iterative multigrid PIV image processing. Experiments in Fluids 29 (1), S051-S060.

Scolan, Y. M., 2004. Hydroelastic behaviour of a conical shell impacting on a quiescent-free surface of an incompressible liquid. Journal of Sound and Vibration 277 (1), 163-203. 
Scolan, Y. M., Korobkin, A., 2001. Three-dimensional theory of water impact. Part 1. Inverse Wagner problem. Journal of Fluid Mechanics 440, 293-326.

Scolan, Y. M., Korobkin, A. A., 2003. Energy distribution from vertical impact of a three-dimensional solid body onto the flat free surface of an ideal fluid. Journal of Fluids and Structures 17 (2), 275-286.

Shams, A., Jalalisendi, M., Porfiri, M., 2015. Experiments on the water entry of asymmetric wedges using particle image velocimetry. Physics of Fluids 27 (2), 027103

Shams, A., Porfiri, M., 2015. Treatment of hydroelastic impact of flexible wedges. Journal of Fluids and Structures, accepted for publication.

Stenius, I., Rosén, A., Battley, M., Allen, T., 2013. Experimental hydroelastic characterization of slamming loaded marine panels. Ocean Engineering $74,1-15$.

Stenius, I., Rosén, A., Kuttenkeuler, J., 2011. Hydroelastic interaction in panel-water impacts of high-speed craft. Ocean Engineering 38 (2), 371-381.

Sun, S. L., Wu, G. X., 2013. Oblique water entry of a cone by a fully three-dimensional nonlinear method. Journal of Fluids and Structures 42 , 313-332.

Tassin, A., Jacques, N., Alaoui, A. E. M., Nême, A., Leblé, B., 2012. Hydrodynamic loads during water impact of three-dimensional solids: Modelling and experiments. Journal of Fluids and Structures 28, 211-231.

Thielicke, W., Stamhuis, E. J., 2014a. PIVlab - Time-Resolved Digital Particle Image Velocimetry Tool for MATLAB (version: 1.32). URL http://dx.doi.org/10.6084/m9.figshare.1092508

Thielicke, W., Stamhuis, E. J., 2014b. PIVlab - Towards user-friendly, affordable and accurate digital particle image velocimetry in MATLAB. Journal of Open Research Software 2 (1), e30.

Tveitnes, T., Fairlie-Clarke, A. C., Varyani, K., 2008. An experimental investigation into the constant velocity water entry of wedge-shaped sections. Ocean Engineering 35 (14), 1463-1478.

Van Oudheusden, B. W., 2013. PIV-based pressure measurement. Measurement Science and Technology 24 (3), 032001.

Von Karman, T., 1929. The impact on seaplane floats, during landing. NACA-TN-321.

Wagner, H., 1932. Uber stoss-und gleitvorgange an der ober- flache von flussigkeite. ZAMM - Zeitschrift fur Ange- wandte Mathematik und Mechanik 12 (4), 193-215.

Zhao, R., Faltinsen, O., 1993. Water entry of two-dimensional bodies. Journal of Fluid Mechanics 246, 593-612. 